\title{
HELICAL IMMERSIONS AND NORMAL SECTIONS
}

\author{
By Yi HONG AND CHORNG-SHI HouH
}

\section{Introduction.}

Let $f: M^{n} \rightarrow \bar{M}^{n+p}$ be an isometric immersion of a connected $n$-dimensional Riemannian manifold $M$ into a Riemannian manifold $\bar{M}$ of dimension $n+p$. If $\gamma: I=[0,1] \rightarrow M$ is a curve on $M$ then $\sigma=f \circ \gamma$ is a curve on $\bar{M}$. Let $\sigma$ be parametrized by its arc length, $\sigma^{(1)}=\dot{\sigma}$ be the unit tangent vector and $K_{1}=$ $\left\|\tilde{\nabla}_{\dot{\sigma}} \sigma^{(1)}\right\| . \tilde{\nabla}$ denotes the covariant differentiation of $\bar{M}$. If $K_{1}$ vanishes on $[0,1]$ then $\sigma$ is called of order 1 . If $K_{1}$ is not identically zero, then we define $\sigma^{(2)}$ by $\tilde{\nabla}_{\dot{\sigma}} \sigma^{(1)}=K_{1} \sigma^{(2)}$ on the set $I_{1}=\left\{s \in[0,1]: K_{1}(s) \neq 0\right\}$. Let $K_{2}=\left\|\tilde{\nabla}_{\dot{\sigma}} \sigma^{(2)}+K_{1} \sigma^{(1)}\right\|$. If $K_{2} \equiv 0$ on $I_{1}$ then $\sigma$ is called of order 2 . If $K_{2}$ is not identically zero on $I_{1}$ then we define $\sigma^{(3)}$ by $\tilde{\nabla}_{\dot{\sigma}} \sigma^{(2)}=-K_{1} \sigma^{(1)}+K_{2} \sigma^{(3)}$. Inductively we put $K_{d}=$ $\left\|\tilde{\nabla}_{\dot{\sigma}} \sigma^{(d)}+K_{d-1} \sigma^{(d-1)}\right\|$. If $K_{d} \equiv 0$ on $I_{d-1}$ then $\sigma$ is called of order $d$. It follows that if the curve $\sigma$ is of order $d$ we have the Frenet formula ([9]):

$$
\tilde{\nabla}_{\dot{\sigma}}\left(\sigma^{(1)}, \sigma^{(2)}, \cdots, \sigma^{(d)}\right)=\left(\sigma^{(1)}, \sigma^{(2)}, \cdots, \sigma^{(d)}\right) K
$$

where

$$
K=\left[\begin{array}{ccccccc}
0 & -K_{1} & 0 & \ldots \ldots \ldots \ldots & 0 \\
K_{1} & 0 & - & K_{2} & & & 0 \\
0 & K_{2} & 0 & \ddots & & \\
& 0 & \ddots & & & \ddots & -K_{d-1} \\
& & & \ddots & K_{d-1} & 0
\end{array}\right]
$$

$K_{1}, K_{2}, \cdots, K_{d-1}$ are called the Frenet curvatures of $\sigma$. If, for each geodesic $\gamma$ on $M$, the curve $f \circ \gamma$ on $\bar{M}$ has constant Frenet curvatures of order $d$, and they are independent of $\gamma$, then $f$ is called a helical immersion of order $d$. In most cases the ambient space is considered as a Riemannian manifold of constant sectional curvature $c$, denoted by $\bar{M}^{n+p}(c)$. Sakamoto [9] and Nakagawa [8] have investigated helical immersions. The concept "helical immersion" originates from Besse [2]; it is important in the theory of harmonic manifolds.

Another important concept used in this paper called normal sections, originated from Chen [3]. In [3], [4], [7], submanifolds in $E^{m}$ with (pointwise) planar normal sections were investigated. Chen and Verheyen [5] proved that

Received March 19, 1984 
a helical submanifold in $E^{m}$ has geodesic normal sections. Verheyen [10] proved its inverse.

For a submanifold $M^{n}$ immersed in a space form $\bar{M}^{n+p}(c)$, we can also define normal sections. For a point $x$ in $M$ and a unit vector $t \in T_{x} M$, the vector $t$ and the normal space $N_{x} M$ determine a $(p+1)$-dimensional subspace $E(x, t)$ of $T_{x} \bar{M}$, which determines a $(p+1)$-dimensional totally geodesic submanifold $M_{0}$. The intersection of $M$ and $M_{0}$ gives rise a curve $\gamma(s)$ (in a neighborhood of $x$ ), called the normal section of $M$ at $x$ in the direction $t$.

For any two vector fields $X, Y$ tangent to $M$, the second fundamental form $h$ is given by $h(X, Y)=\tilde{\nabla}_{X} Y-\nabla_{X} Y$ where $\nabla$ is the covariant differentiation in $M$. For any vector field $\xi$ normal to $M$, put $\tilde{\nabla}_{X} \xi=-A_{\xi} X+\nabla_{X}^{\perp} \xi$, where $-A_{\xi} X$ and $\nabla_{X}^{\frac{1}{X}} \xi$ denote the tangential and normal components of $\tilde{\nabla}_{X} \xi$, respectively.

The covariant differentiation $D$ on the Whitney sum $T(M) \oplus N(M)$ is defined as follows (see [8]): For any $N(M)$-valued tensor field $T$ of type $(1, k), C^{\infty}$-vector fields $X, X_{1}, X_{2}, \cdots, X_{k}$ tangent to $M$, put

$$
\begin{aligned}
& D T\left(X, X_{1}, X_{2}, \cdots, X_{k}\right)=\left(D_{X} T\right)\left(X_{1}, \cdots, X_{k}\right) \\
= & \nabla_{X}^{\perp}\left(T\left(X_{1}, \cdots, X_{k}\right)\right)-\sum_{r=1}^{k} T\left(X_{1}, \cdots, \nabla_{X} X_{r}, \cdots, X_{k}\right) .
\end{aligned}
$$

We have the Ricci identity:

$$
\begin{aligned}
& \left(D^{2} T\right)\left(X, Y, X_{1}, \cdots, X_{k}\right)-\left(D^{2} T\right)\left(Y, X, X_{1}, \cdots, X_{k}\right) \\
= & R^{\perp}(X, Y) T\left(X_{1}, \cdots, X_{k}\right)-\sum_{r=1}^{k} T\left(X_{1}, \cdots, R(X, Y) X_{r}, \cdots, X_{k}\right)
\end{aligned}
$$

where $\left.R^{\perp}(X, Y)=\nabla_{X}^{\frac{1}{X}} \nabla_{Y}^{\frac{1}{Y}}-\nabla_{Y}^{\frac{1}{Y}} \nabla_{X}^{\frac{1}{X}}-\nabla_{[X}^{\perp} X, Y\right]$ is the normal curvature tensor, $R$ is the curvature tensor of $M$.

The following identity is well known $([2])$ :

$$
\left\langle R^{\perp}(X, Y) \xi, \eta\right\rangle=\left\langle\left[A_{\xi}, A_{\eta}\right] X, Y\right\rangle .
$$

The following algebraic Lemma is a main tool in this paper.

Lemma 1.1. Let $T_{1}, T_{2}$ be tensors of $(q, p)$-type on a vector space $V$. Suppose for all $v \in V$

$$
T_{1}\left(v^{p}\right)=T_{1}(v, v, \cdots, v)=T_{2}\left(v^{p}\right),
$$

then for $v_{1}, \cdots, v_{p} \in V$,

$$
\sum_{\sigma \in S_{p}} T_{1}\left(v_{\sigma(1)}, \cdots, v_{\sigma(p)}\right)=\sum_{\sigma \in S_{p}} T_{2}\left(v_{\sigma(1)}, \cdots, v_{\sigma(p)}\right),
$$

where $S_{p}$ is the symmetric group on pletters.

Proof. Let $\lambda_{1}, \cdots, \lambda_{p}$ be real parameters. Take $v=\sum_{i=1}^{p} \lambda_{i} v_{i}$ in (1.5). We have 


$$
\sum \lambda_{\imath_{1}} \lambda_{\imath_{2}} \cdots \lambda_{\imath_{p}} T_{1}\left(v_{i_{1}}, v_{i_{2}}, \cdots, v_{i_{p}}\right)=\sum \lambda_{\imath_{1}} \cdots \lambda_{\imath_{p}} T_{2}\left(v_{i_{1}}, v_{i_{2}}, \cdots, v_{i_{p}}\right) \text {. }
$$

Comparing the coefficients of $\lambda_{1} \cdot \lambda_{2} \cdots \lambda_{p}$ on both sides we have (1.6).

In $\S 2$ we discuss the relation between helical immersion and normal section. In $\S 3$ we consider helical immersed surfaces.

\section{Helical immersions and normal sections.}

The following theorem is a generalization of a theorem of Sakamoto ([9]).

THEOREM 2.1. Let $f$ be an isometric immersion $M^{n} \rightarrow \bar{M}^{n+p}(c)$. For all geodesics $\gamma$ on $M$ suppose $\sigma=f \circ \gamma$ have constant curvatures $K_{1}, K_{2}, \cdots K_{2}(\jmath \leqq d-1$, $d$ the order of $\sigma$ ), then we have the Frenet frames:

$\left(F_{1}\right) \quad \sigma^{(1)}=X$,

$\left(F_{\imath}\right) \quad \sigma^{(i)}=\left(K_{1} \cdots K_{\imath-1}\right)^{-1} \sum_{l=0}^{[i / 2]-1} a_{\imath, \imath-2 l}\left(D^{\imath-2 l-2} h\right)\left(X^{\imath-2 l}\right), \quad 2 \leqq i \leqq \jmath+1$

where $X=\dot{\gamma}, a_{\imath, \imath}=1, \quad a_{\imath, \imath-2 l}=\sum_{\left(i_{1}, \cdots, \imath_{l}\right) \in A_{2}} K_{\imath_{1}}^{2} K_{\imath_{2}}^{2} \cdots K_{\imath_{l}}^{2}$ for $l>0$, where $A_{\imath}$ is the collection of subsets of $\{2,3, \cdots, \imath-2\}$, any two numbers in such subsets have difference at least 2.

Also, for $2 \leqq k, l \leqq 2 \jmath+1,0 \leqq \gamma \leqq k-1, \quad k+l \leqq 2 \jmath+3, \quad X, Y \in U_{x} M$, the unit tangent sphere at $x$,

$$
\begin{aligned}
& \left\langle\left(D^{k-2} h\right)\left(X^{r}, Y, X^{k-r-1}\right),\left(D^{l-2} h\right)\left(X^{l}\right)\right\rangle \\
& = \begin{cases}(-1)^{(k-l) / 2} \nu_{(k+l) / 2}\langle X, Y\rangle, & k+l=\text { even }, \\
0 & k+l=\text { odd } .\end{cases}
\end{aligned}
$$

Where $\nu_{\imath}=\left\|\left(D^{\imath-2} h\right)\left(X^{\imath}\right)\right\|^{2}$ only depend on $K_{1}, \cdots, K_{\imath-1}$, and for $k \leqq 2 \jmath+1$

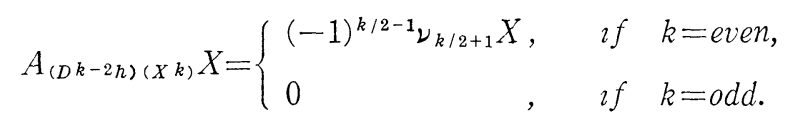

Proof. For $\jmath=1, K_{1}=$ constant implies that $\|h(X, X)\|=K_{1}$ is a constant. So

$$
\langle h(X, X), h(X, Y)\rangle=K_{1}^{2}\langle X, Y\rangle
$$

and $\sigma^{(1)}=X, \sigma^{(2)}=K_{1}^{-1} h(X, X)$. This proves $\left(F_{1}\right),\left(F_{2}\right)$. Also

$$
\begin{aligned}
& \left\langle(D h)\left(X^{2}, Y\right), h\left(X^{2}\right)\right\rangle=1 / 2 Y\left\langle h\left(\tilde{X}^{2}\right), h\left(\tilde{X}^{2}\right)\right\rangle=0, \\
& \left\langle(D h)\left(X^{3}\right), h(X, Y)\right\rangle=-\left\langle h\left(X^{2}\right),(D h)\left(X^{2}, Y\right)\right\rangle=0
\end{aligned}
$$

where $\tilde{X}, \tilde{Y}$ denote the vector fields adapted to $X, Y$, i. e. $\nabla_{X} \tilde{Y}, \nabla_{Y} \tilde{X}, \nabla_{Y} \tilde{Y}$ are 0 .

Suppose the theorem is true for $j-1$. Assume that $K_{1}, \cdots, K_{,}$are constant. 
By inductive hypothesis we have $\left(F_{1}\right),\left(F_{2}\right), \cdots,\left(F_{j}\right)$, also $(2.1)$ for $k+l \leqq 2 j+1$, $2 \leqq k, l \leqq 2 j-1$, (2.2) for $k \leqq 2 j-1$. Then

$$
\begin{aligned}
K_{j} \sigma^{(\jmath+1)}= & \tilde{\nabla}_{X} \sigma^{(j)}+K_{\jmath-1} \sigma^{(\jmath-1)} \\
& =\left(K_{1} \cdots K_{\jmath-1}\right)^{-1} \sum_{l=0}^{[j / 2]-1} a_{\jmath, \jmath-2 l}\left[-A_{\left.\left(D^{\jmath-2 l-2} h\right)\left(X^{\jmath-2 l}\right) X+\left(D^{\jmath-2 l-1} h\right)\left(X^{\jmath-2 l+1}\right)\right]}\right. \\
& +K_{\jmath-1}\left(K_{1} \cdots K_{\jmath-2}\right)^{-1} \sum_{l=0}^{[j-1 / 2]-1} a_{\jmath-1, \jmath-1-2 l}\left(D^{\jmath-3-2 l} h\right)\left(X^{\jmath-1-2 l}\right) .
\end{aligned}
$$

Since $\sigma^{(\jmath+1)}$ is orthogonal to $X$ and $A_{\left(D^{j-2 l-2} h\right)\left(X^{\jmath-2 l}\right)} X \wedge X=0$, we have

$$
K_{j} \sigma^{(\jmath+1)}=\left(K_{1} \cdots K_{\jmath-1}\right)^{-1} \sum_{l=0}^{[j+1 / 2]-1} a_{j+1, j+1-2 l}\left(D^{\jmath-2 l-1} h\right)\left(X^{\jmath-2 l+1}\right)
$$

where $a_{j+1, j+1}=a_{\jmath, j}=1$, and for $l>0$ and $j-2 l>1$

$$
\begin{aligned}
a_{j+1, j+1-2 l} & =K_{J-1}^{2} a_{\jmath-1, j+1-2 l}+a_{\jmath, \jmath-2 l} \\
& =K_{j-1}^{2} \sum_{\left(i_{1}, \cdots, \imath_{l-1} \in A_{\jmath}-1\right.} K_{\imath_{1}}^{2} \cdots K_{\imath_{l-1}}^{2}+\sum_{\left(i_{1}, \cdots, \imath_{l}\right) \in A_{\jmath}} K_{\imath_{1}}^{2} \cdots K_{\imath_{l}}^{2} \\
& =\sum_{\left(i_{1}, \cdots, \imath_{l}\right) \in A_{j+1}} K_{\imath_{1}}^{2} \cdots K_{\imath_{l}}^{2} .
\end{aligned}
$$

If $j$ is odd and $j-2 l=1$ we have $a_{j+1,2}=K_{\jmath-1}^{2} a_{\jmath-1,2}=K_{2}^{2} K_{4}^{2} \cdots K_{j-1}^{2}$. This proves $\left(F_{j+1}\right)$. By $\left\langle\sigma^{(\jmath+1)}, \sigma^{(\jmath+1)}\right\rangle=1$ and $\left(F_{j+1}\right)$ we have

$$
\begin{aligned}
& \left\langle\sum_{l=0}^{[j+1 / 2]-1} a_{\jmath+1, j+1-2 l}\left(D^{\jmath-1-2 l} h\right)\left(X^{\jmath+1-2 l}\right), \sum_{l=0}^{[j+1 / 2]-1} a_{j+1, j+1-2 l}\left(D^{\jmath-1-2 l} h\right)\left(X^{\jmath+1-2 l}\right)\right\rangle \\
& =K_{1}^{2} K_{2}^{2} \cdots K_{\jmath}^{2} .
\end{aligned}
$$

But $\left\langle\left(D^{k-2} h\right)\left(X^{k}\right),\left(D^{\imath-2} h\right)\left(X^{i}\right)\right\rangle$ are constants, depending on $K_{1}, \cdots, K_{\jmath-1}$ for $k+i \leqq 2 j+1$, hence $\nu_{j+1}=\left\langle\left(D^{\jmath-1} h\right)\left(X^{\jmath+1}\right),\left(D^{\jmath-1} h\right)\left(X^{j+1}\right)\right\rangle$ is a constant, depending on $K_{1}, \cdots, K_{\text {. }}$.

For every $l, 2 \leqq l \leqq j+1$

$$
\begin{aligned}
0 & =X\left\langle\left(D^{2 \jmath-1-l} h\right)\left(\tilde{X}^{2 \jmath-l+1}\right),\left(D^{l-2} h\right)\left(\tilde{X}^{l}\right)\right\rangle \\
& =\left\langle\left(D^{2 \jmath-l} h\right)\left(X^{2 \jmath-l+2}\right),\left(D^{l-2} h\right)\left(X^{l}\right)\right\rangle+\left\langle\left(D^{2 \jmath-1-l} h\right)\left(X^{2 \jmath-l+1}\right),\left(D^{l-1} h\right)\left(X^{l+1}\right)\right\rangle .
\end{aligned}
$$

So we have

$$
\left\langle\left(D^{2 \jmath-l} h\right)\left(X^{2 \jmath-l+2}\right),\left(D^{\ell-2} h\right)\left(X^{\iota}\right)\right\rangle=(-1)^{\jmath-l+1} \nu_{j+1}, 2 \leqq l \leqq j+1 .
$$

Again,

$$
\left\langle\left(D^{\jmath} h\right)\left(X^{\jmath+2}\right),\left(D^{\jmath-1} h\right)\left(X^{\jmath+1}\right)\right\rangle=\frac{1}{2} X\left\langle\left(D^{\jmath-1} h\right)\left(\tilde{X}^{\jmath+1}\right),\left(D^{\jmath-1} h\right)\left(\tilde{X}^{\jmath+1}\right)\right\rangle=0 .
$$

But

$$
\begin{aligned}
0 & =X\left\langle\left(D^{2 \jmath-l} h\right)\left(\tilde{X}^{2 \jmath-l+2}\right),\left(D^{l-2} h\right)\left(\tilde{X}^{l}\right)\right\rangle \\
& =\left\langle\left(D^{2 \jmath-l+1} h\right)\left(X^{2 \jmath-l+3}\right),\left(D^{l-2} h\right)\left(X^{l}\right)\right\rangle+\left\langle\left(D^{2 \jmath-l} h\right)\left(X^{2 \jmath-l+2}\right),\left(D^{l-1} h\right)\left(X^{l+1}\right)\right\rangle .
\end{aligned}
$$




\section{Therefore}

$$
\left\langle\left(D^{2 \jmath-l} h\right)\left(X^{2 \jmath-l+2}\right),\left(D^{l-1} h\right)\left(X^{l+1}\right)\right\rangle=0, \quad 1 \leqq l \leqq j .
$$

To prove (2.1) is true for $k+l=2 j+2,2 j+3,2 \leqq k, l \leqq 2 j+1$, by (2.4) and (2.5) we need only to consider the case $\langle X, Y\rangle=0$.

Differentiating

$$
\left\langle\left(D^{2 \jmath-1-l} h\right)\left(\tilde{X}^{r}, \tilde{Y}, \tilde{X}^{2 \jmath-l-r}\right),\left(D^{l-2} h\right)\left(\tilde{X}^{l}\right)\right\rangle=0, \quad 2 \leqq l \leqq 2 j-1,0 \leqq r \leqq 2 j-l
$$

along the directions of $X$ and $Y$ respectively we have

$$
\begin{aligned}
& \left\langle\left(D^{2 \jmath-2} h\right)\left(X^{r}, Y, X^{2 \jmath-r-1}\right), h\left(X^{2}\right)\right\rangle \\
& \quad=-\left\langle\left(D^{2 \jmath-3} h\right)\left(X^{r-1}, Y, X^{2 \jmath-1-r}\right),(D h)\left(X^{3}\right)\right\rangle=\cdots \\
& =(-1)^{r}\left\langle\left(D^{2 \jmath-2-r} h\right)\left(Y, X^{2 \jmath-1-r}\right),\left(D^{r} h\right)\left(X^{r+2}\right)\right\rangle \\
& =(-1)^{r+1}\left\langle\left(D^{2 \jmath-3-r} h\right)\left(X^{2 \jmath-1-r}\right),\left(D^{r+1} h\right)\left(Y, X^{r+2}\right)\right\rangle=\cdots \\
& =\left\langle h\left(X^{2}\right),\left(D^{2 \jmath-2} h\right)\left(X^{2 \jmath-3-r}, Y, X^{r+2}\right)\right\rangle .
\end{aligned}
$$

By Ricci identities for any $4 \leqq k \leqq 2 j+1,2 \leqq l \leqq 2 j-1$,

$$
\begin{aligned}
& \left(D^{k-2} h\right)\left(Y, X^{k-1}\right)-\left(D^{k-2} h\right)\left(X, Y, X^{k-2}\right) \\
& =-R^{\perp}(X, Y)\left(D^{k-4} h\right)\left(X^{k-2}\right)+\sum_{s=0}^{k-3}\left(D^{k-4} h\right)\left(X^{s}, R(X, Y) X, X^{k-3-s}\right) .
\end{aligned}
$$

Since $\langle R(X, Y) X, X\rangle=0$,

$$
\left\langle\left(D^{k-4} h\right)\left(X^{s}, R(X, Y) X, X^{k-3-s}\right),\left(D^{l-2} h\right)\left(X^{l}\right)\right\rangle=0 .
$$

By (2.2)

$$
\begin{aligned}
& \left\langle R^{\perp}(X, Y)\left(D^{k-4} h\right)\left(X^{k-2}\right),\left(D^{l-2} h\right)\left(X^{l}\right)\right\rangle \\
& \quad=\left\langle\left[A_{\left(D^{k-4} h\right)\left(X^{k-2)}\right.}, A_{\left(D^{l-2} h\right)(X l)}\right] X, Y\right\rangle=0 .
\end{aligned}
$$

Hence

$$
\left\langle\left(D^{k-2} h\right)\left(Y, X^{k-1}\right),\left(D^{l-2} h\right)\left(X^{l}\right)\right\rangle=\left\langle\left(D^{k-2} h\right)\left(X, Y, X^{k-2}\right),\left(D^{l-2} h\right)\left(X^{l}\right)\right\rangle
$$

and then

$$
\begin{aligned}
& \left\langle\left(D^{2 \jmath-2-\gamma} h\right)\left(Y, X^{2 \jmath-1-\gamma}\right),\left(D^{\gamma} h\right)\left(X^{\gamma+2}\right)\right\rangle \\
& =\left\langle\left(D^{2 \jmath-2-\gamma} h\right)\left(X, Y, X^{2 \jmath-2-\gamma}\right),\left(D^{\gamma} h\right)\left(X^{\gamma+2}\right)\right\rangle \\
& =-\left\langle\left(D^{2 \jmath-3-\gamma} h\right)\left(Y, X^{2 \jmath-2-\gamma}\right),\left(D^{\gamma+1} h\right)\left(X^{\gamma+3}\right)\right\rangle \\
& =-\left\langle\left(D^{2 \jmath-3-\gamma} h\right)\left(X, Y, X^{2 \jmath-3-\gamma}\right),\left(D^{\gamma+1} h\right)\left(X^{\gamma+3}\right)\right\rangle=\cdots \\
& =(-1)^{2 \jmath-2-\gamma}\left\langle h(X, Y),\left(D^{2 \jmath-2} h\right)\left(X^{2 j}\right)\right\rangle .
\end{aligned}
$$

Thus we have

$$
\left\langle\left(D^{2 \jmath-2} h\right)\left(X^{r}, Y, X^{2 \jmath-1-\gamma}\right), h\left(X^{2}\right)\right\rangle=\left\langle\left(D^{2 \jmath-2} h\right)\left(X^{2 j}\right), h(X, Y)\right\rangle .
$$


On the other hand if we write the identity $\left\langle\left(D^{2 j-2} h\right)\left(X^{2 j}\right), h\left(X^{2}\right)\right\rangle=(-1)^{\jmath+1} \nu_{j+1}$ into the form

$$
\left\langle\left(D^{2 \jmath-2} h\right)\left(X^{2 j}\right), h\left(X^{2}\right)\right\rangle=(-1)^{\jmath+1} \nu_{j+1}(\langle X, X\rangle)^{\jmath+1},
$$

by Lemma 1.1 we have

$$
\sum_{\gamma=0}^{2 \jmath-1}\left\langle\left(D^{2 \jmath-2} h\right)\left(X^{\gamma}, Y, X^{2 \jmath-\gamma-1}\right), h\left(X^{2}\right)\right\rangle+2\left\langle\left(D^{2 \jmath-2} h\right)\left(X^{2 \jmath}\right), h(X, Y)\right\rangle=0 .
$$

Hence we have

$$
\left\langle\left(D^{2 \jmath-2} h\right)\left(X^{\gamma}, Y, X^{2 \jmath-\gamma-1}\right), h\left(X^{2}\right)\right\rangle=\left\langle\left(D^{2 \jmath-2} h\right)\left(X^{2 j}\right), h(X, Y)\right\rangle=0 .
$$

This shows that for $0 \leqq s \leqq 2 j-2,0 \leqq \gamma \leqq 2 j-1-s$

$$
\left\langle\left(D^{2 \jmath-2-s} h\right)\left(X^{r}, Y, X^{2 \jmath-1-s-r}\right),\left(D^{s} h\right)\left(X^{s+2}\right)\right\rangle=0 .
$$

Now

$$
\begin{aligned}
&\left\langle\left(D^{2 \jmath-1} h\right)\left(X^{\gamma}, Y, X^{2 \jmath-\gamma}\right), h\left(X^{2}\right)\right\rangle \\
&= X\left\langle\left(D^{2 \jmath-2} h\right)\left(\tilde{X}^{\gamma-1}, \tilde{Y}, \tilde{X}^{2 \jmath-\gamma}\right), h\left(\tilde{X}^{2}\right)\right\rangle \\
&-\left\langle\left(D^{2 \jmath-2} h\right)\left(X^{\gamma-1}, Y, X^{2 \jmath-\gamma}\right),(D h)\left(X^{3}\right)\right\rangle \\
&=-\left\langle\left(D^{2 \jmath-2} h\right)\left(X^{\gamma-1}, Y, X^{2 \jmath-\gamma}\right),(D h)\left(X^{3}\right)\right\rangle=\cdots \\
&=(-1)^{\gamma}\left\langle\left(D^{2 \jmath-\gamma-1} h\right)\left(Y, X^{2 \jmath-\gamma}\right),\left(D^{\gamma} h\right)\left(X^{\gamma+2}\right)\right\rangle \\
&=(-1)^{\gamma}\left\langle\left(D^{2 \jmath-\gamma-1} h\right)\left(X, Y, X^{2 \jmath-\gamma-1}\right),\left(D^{\gamma} h\right)\left(X^{\gamma+2}\right)\right\rangle \\
&=(-1)^{\gamma+1}\left\langle\left(D^{2 \jmath-\gamma-2} h\right)\left(Y, X^{2 \jmath-\gamma-1}\right),\left(D^{\gamma+1} h\right)\left(X^{\gamma+3}\right)\right\rangle=\cdots \\
&=-\left\langle h(X, Y),\left(D^{2 \jmath-1} h\right)\left(X^{2 \jmath+1}\right)\right\rangle .
\end{aligned}
$$

By (2.5) and Lemma 1.1 we have

$$
\sum_{\gamma=0}^{2 \jmath}\left\langle\left(D^{2 \jmath-1} h\right)\left(X^{\gamma}, Y, X^{2 \jmath-r}\right), h\left(X^{2}\right)\right\rangle+2\left\langle\left(D^{2 \jmath-1} h\right)\left(X^{2 \jmath+1}\right), h(X, Y)\right\rangle=0 .
$$

Since $j>1$,

$$
\left\langle\left(D^{2 \jmath-1} h\right)\left(X^{\gamma}, Y, X^{2 \jmath-r}\right), h\left(X^{2}\right)\right\rangle=\left\langle\left(D^{2 \jmath-1} h\right)\left(X^{2 \jmath+1}\right), h(X, Y)\right\rangle=0
$$

and for $0 \leqq s \leqq 2 j-2,0 \leqq \gamma \leqq 2 j-1-s$,

$$
\left\langle\left(D^{2 \jmath-1-s} h\right)\left(X^{r}, Y, X^{2 \jmath-s-r}\right),\left(D^{s} h\right)\left(X^{s+2}\right)\right\rangle=0 .
$$

This proves (2.1) for $k+l=2 j+2,2 j+3$. (2.2) is a consequence of (2.1).

Remark. In proving $\left(F_{0+1}\right),(2.4)$ and (2.8) we only need the assumption that $K_{3}$ is a function of the point $x$, not depending on the direction $X$.

COROLLARY 2.2. If for every geodesic $\gamma$ the Frenet curvatures $K_{1}, \cdots, K_{\text {, of }}$ 
$\boldsymbol{\sigma}=f \circ \gamma$ are constants, then $\boldsymbol{\sigma}^{(2)}, \cdots, \boldsymbol{\sigma}^{(\jmath+1)} \in N_{x} M$. Especially if $K_{1}, \cdots, K_{d-1}$ are constants then $f$ is an immersion with geodesic normal sections.

Proof. The first conclusion follows from theorem 2.1. For the second conclusion assume $K_{1}, \cdots, K_{d-1}$ are constants then $\sigma^{(2)}, \cdots, \sigma^{(d)} \in N_{x} M$. By the theory of ordinary differential equations we know that the geodesic $\gamma$ is contained in the totally geodesic submanifold $M_{0}$, whose tangent space at $x$ is spanned by $\sigma^{(1)}, \sigma^{(2)}, \cdots, \sigma^{(d)}$, which is contained in $E(x, X)$. This means $\sigma$ is a normal section of $M$ at $x$ in the direction $X$.

The second assertion, i.e. a helical submanifold has geodesic normal sections was proved by Chen and Verheyen ([5]). The inverse of theorem 2.1 is also true.

THEOREM 2.3. Let $f: M^{n} \rightarrow \bar{M}^{n+p}(c)$ be an isometric immersion, $j \geqq 1$. If at

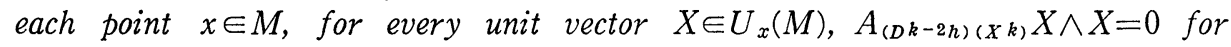
$2 \leqq k \leqq 2 j+1$, then the Frenet curvatures $K_{1}, K_{2}, \cdots, K$, are constants, and so (2.1), (2.2) hold.

Proof. If $A_{h\left(X^{2}\right)} X=\mu_{1} X$ holds for some $\mu_{1}$ ( $\mu_{1}$ may depend on $\left.X\right)$, then for $Y \in U_{x} M,\left\langle h\left(X^{2}\right), h(X, Y)\right\rangle=\mu_{1}\langle X, Y\rangle$. This implies that $K_{1}^{2}=\left\|h\left(X^{2}\right)\right\|^{2}=\mu_{1}$ is constant on $U_{x} M$. By the assumption $A_{(D h)\left(X^{3}\right)} X=\mu_{2} X$ for some $\mu_{2}$. So for $Y \in U_{x}(M)$,

$$
\begin{aligned}
& \left\langle(D h)\left(X^{3}\right), h(X, Y)\right\rangle=\mu_{2}\langle X, Y\rangle \\
& \left\langle(D h)\left(X^{3}\right), h(X, Y)\right\rangle=X\left\langle h\left(\tilde{X}^{2}\right), h(\tilde{X}, \tilde{Y})\right\rangle-\left\langle h\left(X^{2}\right),(D h)\left(X^{2}, Y\right)\right\rangle \\
& =\left(X \mu_{1}\right)\langle X, Y\rangle-1 / 2 Y\left\langle h\left(\tilde{X}^{2}\right), h\left(\tilde{X}^{2}\right)\right\rangle=\left(X \mu_{1}\right)\langle X, Y\rangle-1 / 2 Y \mu_{1} .
\end{aligned}
$$

Since $Y$ is arbitrary and $X$ can be chosen such that $\langle X, Y\rangle=0$ we see that $Y \mu_{1}=0, \mu_{1}$ is a constant on $M$. By Lemma 1.1, we also have

$$
\left\langle(D h)\left(X^{2}, Y\right), h\left(X^{2}\right)\right\rangle=0 .
$$

It is easy to see $\mu_{2}=0$. This proves the theorem in case $j=1$.

Suppose the theorem is true for $j-1$. Assume that $A_{(D k-2 h)\left(X^{k}\right)} X=\mu_{k-1} X$ for $2 \leqq k \leqq 2 j+1, X \in U_{x} M$. By inductive hypothesis, $K_{1}, \cdots, K_{\jmath-1}$ are constants and so $\mu_{1}, \mu_{2}, \cdots, \mu_{2,-2}$ are constants. By differentiating the identity (when $\left.Y \in U_{x} M\right)$ :

$$
\left\langle\left(D^{2 \jmath-3} h\right)\left(\tilde{X}^{2 \jmath-1}\right), h(\tilde{X}, \tilde{Y})\right\rangle=0
$$

along the direction of $X$ we have

$$
\left\langle\left(D^{2 \jmath-3} h\right)\left(X^{2 \jmath-1}\right),(D h)\left(X^{2}, Y\right)\right\rangle=-\mu_{2 \jmath-1}\langle X, Y\rangle .
$$

Suppose we have proved that for $0 \leqq \gamma \leqq i-1,2 \leqq i \leqq k, k \leqq 2 j-2$

$$
\left\langle\left(D^{\imath-2} h\right)\left(X^{r}, Y, X^{i-1-\gamma}\right),\left(D^{2 \jmath-\imath} h\right)\left(X^{2 \jmath-\imath+2}\right)\right\rangle=(-1)^{\imath} \mu_{2 \jmath-1}\langle X, Y\rangle .
$$


Then by differentiating

$$
\left\langle\left(D^{k-2} h\right)\left(\tilde{X}^{\gamma}, \tilde{Y}, \tilde{X}^{k-1-\gamma}\right),\left(D^{2 \jmath-1-k} h\right)\left(\tilde{X}^{2 \jmath-k+1}\right)\right\rangle=0
$$

along the direction of $X$ we have

$$
\begin{aligned}
& \left\langle\left(D^{k-1} h\right)\left(X^{\gamma+1}, Y, X^{k-1-\gamma}\right),\left(D^{2 \jmath-1-k} h\right)\left(X^{2 \jmath-k+1}\right)\right\rangle \\
& \quad+\left\langle\left(D^{k-2} h\right)\left(X^{\gamma}, Y, X^{k-\gamma-1}\right),\left(D^{2 \jmath-k} h\right)\left(X^{2 \jmath-k+2}\right)\right\rangle=0
\end{aligned}
$$

and thus

$$
\left\langle\left(D^{k-1} h\right)\left(X^{\gamma+1}, Y, X^{k-\gamma-1}\right),\left(D^{2 \jmath-1-k} h\right)\left(X^{2 \jmath-k+1}\right)\right\rangle=(-1)^{k+1} \mu_{2 \jmath-1}\langle X, Y\rangle
$$

for $0 \leqq \gamma \leqq k-1$. Besides by Ricci identity

$$
\begin{aligned}
& \left(D^{k-1} h\right)\left(X, Y, X^{k-1}\right)-\left(D^{k-1} h\right)\left(Y, X^{k}\right) \\
& \quad=R^{\perp}(X, Y)\left(D^{k-3} h\right)\left(X^{k-1}\right)-\sum_{s=0}^{k-2}\left(D^{k-3} h\right)\left(X^{s}, R(X, Y) X, X^{k-s-2}\right)
\end{aligned}
$$

Using the same argument as before we see

$$
\begin{gathered}
\left\langle\left(D^{k-1} h\right)\left(X, Y, X^{k-1}\right),\left(D^{2 \jmath-k-1} h\right)\left(X^{2 \jmath-k+1}\right)\right\rangle \\
=\left\langle\left(D^{k-1} h\right)\left(Y, X^{k}\right),\left(D^{2 \jmath-k-1} h\right)\left(X^{2 \jmath-k+1}\right)\right\rangle .
\end{gathered}
$$

This completes the induction of (2.11). Especially for $Y=X$ and $k=j$ we have

$$
\nu_{j+1}=\left\|\left(D^{\jmath-1} h\right)\left(X^{\jmath+1}\right)\right\|^{2}=(-1)^{\jmath+1} \mu_{2 \jmath-1} .
$$

Now let $X, Y \in U_{x} M$ we can choose $Z \in U_{x} M$ such that $\langle X, Z\rangle=0$ and for some $\alpha \in[0,2 \pi], Y=X \cos \alpha+Z \sin \alpha$. For $t \in[0,2 \pi]$ let $Y_{t}=X \cos t+Z \sin t$ then

$$
\begin{aligned}
& \frac{d}{d t}\left\langle\left(D^{\jmath-1} h\right)\left(Y_{t}^{\jmath+1}\right),\left(D^{\jmath-1} h\right)\left(Y_{t}^{\jmath+1}\right)\right\rangle \\
& \quad=2 \sum_{\gamma=0}^{\jmath}\left\langle\left(D^{\jmath-1} h\right)\left(Y_{t}^{\gamma},-X \sin t+Z \cos t, Y_{t}^{\jmath-\gamma}\right),\left(D^{\jmath-1} h\right)\left(Y_{t}^{\jmath+1}\right)\right\rangle \\
& \quad=2(-1)^{\jmath+1} \mu_{2 \jmath-1}(j+1)\left\langle Y_{t},-X \sin t+Z \cos t\right\rangle=0
\end{aligned}
$$

Hence $\left\|\left(D^{\jmath-1} h\right)\left(Y_{t}^{\jmath+1}\right)\right\|^{2}$ is constant for $t \in[0,2 \pi]$, so

$$
\left\|\left(D^{\jmath-1} h\right)\left(X^{\jmath+1}\right)\right\|=\left\|\left(D^{\jmath-1} h\right)\left(Y^{\jmath+1}\right)\right\| .
$$

This proves $\mu_{2 j-1}$ and $\nu_{j+1}$ are constant on $U_{x} M$.

Now for any $X, Y \in U_{x} M$ with $\langle X, Y\rangle=0$ we have

$$
\left\langle\left(D^{2 j-1} h\right)\left(X^{2 \jmath+1}\right), h(X, Y)\right\rangle=\mu_{2 j}\langle X, Y\rangle=0
$$

and 


$$
\begin{aligned}
& \left\langle\left(D^{2 \jmath-2} h\right)\left(X^{2 j}\right),(D h)\left(X^{2}, Y\right)\right\rangle \\
& \quad=X\left\langle\left(D^{2 \jmath-2} h\right)\left(\tilde{X}^{2 j}\right), h(\tilde{X}, \tilde{Y})\right\rangle-\left\langle\left(D^{2 \jmath-1} h\right)\left(X^{2 \jmath+1}\right), h(X, Y)\right\rangle \\
& =\left(X \mu_{2 \jmath-1}\right)\langle X, Y\rangle=0 .
\end{aligned}
$$

Suppose we have proved that for $1 \leqq q \leqq 2 j-2,0 \leqq \gamma \leqq q, 0 \leqq s \leqq \gamma+1$

$$
\left\langle\left(D^{2 \jmath-1-\gamma} h\right)\left(X^{2 \jmath-\gamma+1}\right),\left(D^{\gamma} h\right)\left(X^{s}, Y, X^{\gamma+1-s}\right)\right\rangle=0,
$$

then for $0 \leqq s \leqq q+1$,

Again, since

$$
\begin{aligned}
0= & \left\langle\left(D^{2 \jmath-1-q} h\right)\left(X^{2 \jmath-q+1}\right),\left(D^{q} h\right)\left(X^{s}, Y, X^{q+1-s}\right)\right\rangle \\
= & X\left\langle\left(D^{2 \jmath-2-q} h\right)\left(\tilde{X}^{2 \jmath-q}\right),\left(D^{q} h\right)\left(\tilde{X}^{s}, \tilde{Y}, \tilde{X}^{q+1-s}\right)\right\rangle \\
& -\left\langle\left(D^{2 \jmath-2-q} h\right)\left(X^{2 \jmath-q}\right),\left(D^{q+1} h\right)\left(X^{s+1}, Y, X^{q+1-s}\right)\right\rangle \\
= & -\left\langle\left(D^{2 \jmath-2-q} h\right)\left(X^{2 \jmath-q}\right),\left(D^{q+1} h\right)\left(X^{s+1}, Y, X^{q+1-s}\right)\right\rangle .
\end{aligned}
$$

$$
\begin{aligned}
& \left(D^{q+1} h\right)\left(X, Y, X^{q+1}\right)-\left(D^{q+1} h\right)\left(Y, X^{q+2}\right) \\
& \quad=R^{\perp}(X, Y)\left(D^{q-1} h\right)\left(X^{q+1}\right)-\sum_{\gamma=0}^{q}\left(D^{q-1} h\right)\left(X^{\gamma}, R(X, Y) X, X^{q-\gamma}\right),
\end{aligned}
$$

as before we can show that

$$
\begin{aligned}
& \left\langle\left(D^{2 \jmath-2-q} h\right)\left(X^{2 \jmath-q}\right),\left(D^{q+1} h\right)\left(Y, X^{q+2}\right)\right\rangle \\
& \quad=\left\langle\left(D^{2 \jmath-2-q} h\right)\left(X^{2 \jmath-q}\right),\left(D^{q+1} h\right)\left(X, Y, X^{q+1}\right)\right\rangle=0 .
\end{aligned}
$$

This completes the induction of (2.13). Putting $\gamma=j$ we have

hence we obtain

$$
\left\langle\left(D^{\jmath-1} h\right)\left(X^{\jmath+1}\right),\left(D^{\jmath} h\right)\left(Y, X^{j+1}\right)\right\rangle=0,
$$

$$
\begin{aligned}
Y \mu_{2 \jmath-1} & =(-1)^{\jmath+1} Y\left\langle\left(D^{\jmath-1} h\right)\left(\tilde{X}^{\jmath+1}\right),\left(D^{\jmath-1} h\right)\left(X^{\jmath+1}\right)\right\rangle \\
& =2(-1)^{j+1}\left\langle\left(D^{\jmath-1} h\right)\left(X^{\jmath+1}\right),\left(D^{\jmath} h\right)\left(Y, X^{j+1}\right)\right\rangle=0,
\end{aligned}
$$

it proves that $\mu_{2 j-1}$ (and also $\nu_{j+1}$ ) is a constant on $M$. It follows that $\mu_{2 j}=0$. Thus by (2.10)-(2.13) we have (2.1). By inductive hypothesis $K_{1}, \cdots, K_{\jmath-1}$ are constants and $\left(F_{1}\right), \cdots,\left(F_{\jmath}\right)$ hold. As in Theorem 2.1 we have

$$
\begin{aligned}
K_{\jmath} \sigma^{(\jmath+1)} & =\tilde{\nabla}_{X} \sigma^{(j)}+K_{\jmath-1} \sigma^{(\jmath-1)} \\
& =\left(K_{1}, \cdots, K_{\jmath-1}\right)^{-1} \sum a_{j+1, j+1-2 l}\left(D^{\jmath-1-2 l} h\right)\left(X^{\jmath+1-2 l}\right)
\end{aligned}
$$

where $a_{j+1, j+1-2 l}$ are constants depending on $K_{1}, \cdots, K_{\jmath-1}$. Since for $\gamma+s \leqq 2 \jmath-2$ $\left\langle\left(D^{r} h\right)\left(X^{r+2}\right),\left(D^{s} h\right)\left(X^{s+2}\right)\right\rangle$ are all constants we see that $K$, must be a constant. The theorem is proved.

COROLlaRY 2.4. An ısometruc immersion $f: M^{n} \rightarrow \bar{M}^{n+p}(c)$ is a helical immer- 
sion if and only if $M$ has geodesic normal sections.

Proof. If every geodesic $\gamma$ is a normal section, i.e. contained in a totally geodesic submanifold $M_{0}$ with $T_{x} M_{0}=E(x, X), X=\dot{\gamma}$, then $X \in T_{x}\left(M_{0}\right), \tilde{\nabla}_{X} X=$ $h(X, X) \in T_{x} M_{0}$ and $\tilde{\nabla}_{X} h(X, X) \in T_{x} M_{0}, \cdots, \tilde{\nabla}_{X}\left(D^{\imath-2} h\right)\left(X^{i}\right) \in T_{x} M_{0}$ for all $i$. This

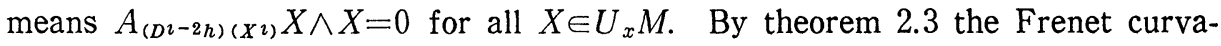
tures are all constants.

COROLLARY 2.5. Let $f: M^{n} \rightarrow \bar{M}^{n+p}(c)$ be an isometric immersion with every geodesic being of order $d$. Then:

(a) If $d$ is even and the Frenet curvatures $K_{1}, K_{2}, \cdots, K_{(d / 2)-1}$ are constants then $f$ is helical, $K_{1}, K_{2}, \cdots, K_{d-1}$ are constants.

(b) If $d$ is odd and the Frenet curvatures $K_{1}, K_{2}, \cdots, K_{(d-3) / 2}$ are constants and $K_{(d-1) / 2}$ is constant on every unit sphere $U_{x} M$ then $f$ is helical.

Proof. (a). If $K_{1}, K_{2}, \cdots, K_{(d / 2)-1}$ are constants by Theorem 2.1 for $k \leqq d-1$,

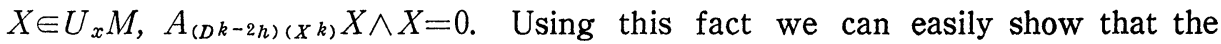
Frenet frames $\sigma^{(1)}, \sigma^{(2)}, \cdots, \sigma^{(d)}$, where $\sigma=f \circ \gamma, \gamma$ being the geodesic issued from $x$ and tangent to $X$, are linear combinations of $X, h\left(X^{2}\right), \cdots,\left(D^{d-2} h\right)\left(X^{d}\right)$. Hence $\sigma^{(1)}, \sigma^{(2)}, \cdots, \sigma^{(d)} \in E(x, X)$. By the theory of differential equations $\sigma$ is contained in the totally geodesic submanifold $M_{0}$ having $E(x, X)$ as tangent space at $x$. Thus $\gamma$ is a geodesic normal section and $f$ is helical.

(b). By the remark after Theorem 2.1 and the same argument in (a) $f$ is also helical.

Chen and Verheyen proved this corollary in the case $d=3,4$. ([5]). Also see Nakagawa [8].

Next we consider some problems related to the order $d$.

THEOREM 2.6. Let $M$ be a compact submanifold in $E^{m}$ having geodesic normal sections, then the order of $M$ is even.

Proof. By Corollary 4 in [5] the geodesics on $M$ are closed curves. But these curves are helices and a helix of odd order in $E^{m}$ cannot be closed. See D. Ferus and S. Schirrmacher [6].

THEOREM 2.7. Let $M$ be a spherical submanifold in $E^{n+p}$ having geodesic normal sections then the order of $M$ is even (in $E^{n+p}$ ).

Proof. Suppose a geodesic $\gamma$ of $M$ is of odd order $2 m+1$. Then there are constants $\gamma_{0}, \gamma_{1}, \cdots, \gamma_{m}, a_{1}, \cdots, a_{m}$ and orthogonal vectors $e_{0}, e_{1}, \cdots, e_{2 m} \in E^{n+p}$ such that

$$
\gamma(t)=\gamma_{0} t e_{0}+\sum_{i=1}^{m} \gamma_{i}\left[e_{2 \imath-1} \cos a_{j} t+e_{2 \imath} \sin a_{j} t\right] .
$$

Since $M$ is contained in some sphere with center $x$, 


$$
\left(\gamma_{0} t-x_{0}\right)^{2}+\sum_{i=1}^{m}\left[\left(\gamma_{2} \cos a_{i} t-x_{22-1}\right)^{2}+\left(\gamma_{\imath} \sin a_{i} t-x_{2 \imath}\right)^{2}\right]=R^{2}
$$

where $x_{k}=\left\langle x, e_{k}\right\rangle, R$ is a constant. But this implies that $\gamma_{0}=0$ which is a contradiction.

In order to classify helical submanifolds in spaces of constant curvature, an important problem is to determine the upper bound of the dimension of the ambient spaces. By Sakamoto [9] a helical submanifold $M$ immersed into a sphere is also a helical submanifold of a Euclidean space. By the proposition 5.6 in Sakamoto [9] we know that if $M^{n} \subset E^{m}$ is a helical submanifold of order $d$ then $M^{n}$ is contained in the linear subspace

$$
O_{x}^{d}=S p\left\{X,\left(D^{k-2} h\right)\left(X_{1}, \cdots, X_{k}\right) ; X, X_{1}, \cdots, X_{k} \in T_{x} M, k=2,3, \cdots, d\right\} .
$$

We have

LEMMA 2.8. Let $M^{n} \subset \tilde{M}^{n+p}(c)$ be an immersion. Then for $j \geqq 1$

$$
\operatorname{dim} O_{x}^{\jmath} \leqq\left(\begin{array}{c}
n+j \\
j
\end{array}\right)-1
$$

Proof. For $\jmath=1$ we have

$$
O_{x}^{1}=S p\left\{X, X \in T_{x} M\right\}=T_{x} M .
$$

So $\operatorname{dim} O_{x}^{1}=n$. Suppose that we have $\operatorname{dim} O_{x}^{\jmath-1} \leqq\left(\begin{array}{c}n+j-1 \\ j-1\end{array}\right)-1$. Noticing that $O_{x}^{j}=S p\left\{O_{x}^{j-1}, V\right\}$ where

$$
V=S p\left\{\left(D^{\jmath-2} h\right)\left(X_{1}, \cdots, X_{j}\right) ; X_{1}, \cdots, X_{j} \in T_{x} M\right\},
$$

if we can show that (where $e_{1}, \cdots, e_{n}$ are basis vectors for $T_{x} M$ )

$$
V \subset S p\left\{\left(D^{\jmath-2} h\right)\left(e_{1}^{k_{1}}, e_{2}^{k_{2}}, \cdots, e_{n}^{k n}\right) ; k_{1}+k_{2}+\cdots+k_{n}=j ; O_{x}^{\jmath-1}\right\},
$$

since there are $\left(\begin{array}{c}n+j-1 \\ j\end{array}\right)$ vectors in the set $\left\{\left(D^{j-2} h\right)\left(e_{1}^{k_{1}}, e_{2}^{k_{2}}, \cdots, e^{k_{n}}\right)\right\}$, then we have $\operatorname{dim} O_{x}^{\jmath} \leqq\left(\begin{array}{c}n+\jmath-1 \\ \jmath\end{array}\right)+\left(\begin{array}{c}n+\jmath-1 \\ j-1\end{array}\right)-1=\left(\begin{array}{c}n+\jmath \\ \jmath\end{array}\right)-1$.

To prove (2.14) notice that

$$
V=S p\left\{\left(D^{j-2} h\right)\left(e_{\imath_{1}}, e_{\imath_{2}}, \cdots, e_{\imath_{j}}\right) ; i_{1}, \imath_{2}, \cdots, \imath_{j}=1,2, \cdots, n\right\} .
$$

We only need to show that for any $\gamma, 1 \leqq \gamma \leqq \jmath-3$,

$$
\begin{aligned}
& \left.\left(D^{\jmath-2} h\right)\left(e_{\imath_{1}}, e_{\imath_{2}}, \cdots, e_{\imath_{\gamma}}, e_{\imath_{\gamma+1}}, \cdots, e_{\imath_{j}}\right)\right) \\
& \quad-\left(D^{j-2} h\right)\left(e_{\imath_{1}}, \cdots, e_{\imath_{\gamma+1}}, e_{\imath_{\gamma}}, \cdots, e_{\imath_{j}}\right) \in O_{x}^{\jmath-1} .
\end{aligned}
$$

Extending $e_{1}, \cdots, e_{n}$ to vector fields $\tilde{e}_{1}, \cdots, \tilde{e}_{n}$ in a neighborhood of $x$ such that at $x, \nabla_{e_{i}} \tilde{e}_{k}=0$ for all $i, k$, 


$$
\begin{aligned}
& \left(D^{\jmath-2} h\right)\left(e_{i_{1}}, e_{\imath_{2}}, \cdots, e_{\imath_{\gamma}}, e_{\imath_{\gamma+1}}, \cdots, e_{\imath_{\jmath}}\right) \\
& -\left(D^{\jmath-2} h\right)\left(e_{\imath_{1}}, \cdots, e_{\imath_{\gamma+1}}, e_{\imath_{\gamma}}, \cdots, e_{\imath_{j}}\right) \\
& =\nabla^{\perp}{ }_{e_{i_{1}}} \nabla^{\perp}{ }_{\tilde{e}_{i_{2}}} \cdots \nabla^{\perp}{ }_{\tilde{e}_{i_{\gamma-1}}}\left[\left(D^{\jmath-\gamma-1} h\right)\left(\tilde{e}_{\imath_{\gamma}}, \tilde{e}_{i_{\gamma+1}}, \cdots, \tilde{e}_{\imath_{j}}\right)\right. \\
& \left.-\left(D^{\jmath-\gamma-1} h\right)\left(\tilde{e}_{\imath_{\gamma+1}}, \tilde{e}_{\imath_{\gamma}}, \cdots, \tilde{e}_{\imath_{j}}\right)\right] \\
& =\nabla^{\perp}{ }_{e_{i_{1}}} \nabla^{\perp}{ }_{\tilde{e}_{i_{2}}} \cdots \nabla^{\perp}{ }_{\tilde{e}_{i_{\gamma-1}}}\left[R^{\perp}\left(\tilde{e}_{i_{\gamma}}, \tilde{e}_{\imath_{\gamma+1}}\right)\left(D^{\jmath-\gamma-3} h\right)\left(\tilde{e}_{\gamma_{\gamma+2}}, \cdots, \tilde{e}_{i_{j}}\right)\right. \\
& \left.-\sum_{s=\gamma+2}^{J}\left(D^{\jmath-\gamma-3} h\right)\left(\tilde{e}_{i_{\gamma+2}}, \cdots, R\left(\tilde{e}_{\imath_{\gamma}}, \tilde{e}_{i_{\gamma+1}}\right) \tilde{e}_{i_{s}}, \cdots, \tilde{e}_{\imath_{j}}\right)\right] \text {. }
\end{aligned}
$$

But $R^{\perp}\left(\tilde{e}_{\imath_{\gamma}}, \tilde{e}_{i_{\gamma+1}}\right)\left(D^{l-\gamma-3} h\right)\left(\tilde{e}_{\imath_{\gamma+2}}, \cdots, \tilde{e}_{\imath_{j}}\right)$ is a linear combination of $h\left(\tilde{e}_{\imath}, \tilde{e}_{l}\right)$, $1 \leqq i, l \leqq n$, in fact if $\xi$ is orthogonal to all $h\left(\tilde{e}_{\imath}, \tilde{e}_{l}\right), 1 \leqq i, l \leqq n$, then

$$
\left\langle A_{\xi} \tilde{e}_{\imath}, \tilde{e}_{l}\right\rangle=\left\langle\xi, h\left(\tilde{e}_{\imath}, \tilde{e}_{l}\right)\right\rangle=0,
$$

hence $A_{\xi}=0$ and $\left\langle R^{\perp}\left(\tilde{e}_{i_{\gamma}}, \tilde{e}_{i_{\gamma+1}}\right) \eta, \xi\right\rangle=\left\langle\left[A_{\eta}, A_{\xi}\right] \tilde{e}_{\imath_{\gamma}}, \tilde{e}_{i_{\gamma+1}}\right\rangle=0$ for all $\eta \in N_{x} M$. Thus all terms in the last expression are in $O_{x}^{j-1}$. This proves (2.15).

Thus we have

THEOREM 2.9. Let $M^{n} \subset E^{m}$ be a helical immersion of order $d$ then $M^{n}$ is contained in a linear subspace $V$ of $E^{m}$ with $\operatorname{dim} V \leqq\left(\begin{array}{c}n+d \\ d\end{array}\right)-1$.

\section{$\S 3$. Surface with geodesic normal sections.}

Chen and Verheyen [5] studied surfaces with geodesic normal sections, they showed that in $E^{5}$ the only surfaces with geodesic normal sections are (i) a 2 -plane $E^{2}$; (ii) an ordinary 2-sphere in a 3-plane; (iii) the Veronese surfaces in $E^{5}$. They also gave some partial results in $E^{6}$.

In this section we will prove the following theorems.

THEOREM 3.1. Let $M^{2}$ be a surface with constant curvature immersed in $E^{7}$, then $M^{2}$ has geodesic normal sections if and only of $M$ is contained in one of the followings.

(i) a 2-plane $E^{2}$;

(ii) an ordinary 2-sphere in a 3-plane;

(iii) the Veronese surface in a 5-plane;

(iv) the 3rd standard immersion of a 2-sphere $S^{2} \subset E^{7}$.

THEOREM 3.2. There is no surface $M^{2}$ helically immersed into $E^{m}$ of order 3 .

First we prove the following lemma.

LEMMA 3.3. Let $M^{2} \subset E^{m}$ be a helical immersion, $\left\{e_{1}, e_{2}\right\}$ are orthonormal vectors in $T_{x} M, x \in M . \quad \beta=\left\|h\left(e_{1}, e_{2}\right)\right\|$. Then

$$
\left\langle(D h)\left(e_{1}^{3}\right), h\left(e_{1}^{2}\right)\right\rangle=\left\langle(D h)\left(e_{1}^{3}\right), h\left(e_{1}, e_{2}\right)\right\rangle=\left\langle(D h)\left(e_{1}^{2}, e_{2}\right), h\left(e_{1}^{2}\right)\right\rangle=0,
$$




$$
\begin{aligned}
& \left\langle(D h)\left(e_{1}^{3}\right), h\left(e_{2}^{2}\right)\right\rangle=-3 \beta e_{1} \beta, \\
& \left\langle(D h)\left(e_{1}^{2}, e_{2}\right), h\left(e_{1}, e_{2}\right)\right\rangle=\beta e_{1} \beta, \\
& \left\langle(D h)\left(e_{1}^{2}, e_{2}\right), h\left(e_{2}^{2}\right)\right\rangle=-\beta e_{2} \beta .
\end{aligned}
$$

Proof. (3.1) is proved in theorem 2.1. Using Lemma (1.1) to $\left\langle(D h)\left(e_{1}^{3}\right), h\left(e_{1}^{2}\right)\right\rangle$ $=0$ we have

$$
\left\langle(D h)\left(e_{1}^{3}\right), h\left(e_{2}^{2}\right)\right\rangle+6\left\langle(D h)\left(e_{1}^{2}, e_{2}\right), h\left(e_{1}, e_{2}\right)\right\rangle+3\left\langle(D h)\left(e_{1}, e_{2}^{2}\right), h\left(e_{1}^{2}\right)\right\rangle=0
$$

and

$$
\begin{aligned}
& \left\langle(D h)\left(e_{1}^{3}\right), h\left(e_{2}^{2}\right)\right\rangle+\left\langle(D h)\left(e_{2}^{2}, e_{1}\right), h\left(e_{1}^{2}\right)\right\rangle \\
& \quad=e_{1}\left\langle h\left(\tilde{e}_{1}^{2}\right), h\left(\tilde{e}_{2}^{2}\right)\right\rangle=e_{1}\left(K_{1}^{2}-2 \beta^{2}\right)=-4 \beta e_{1} \beta,
\end{aligned}
$$

where $\tilde{e}_{1}, \tilde{e}_{2}$ denote the vector fields adapted to $e_{1}, e_{2}$ and

$$
\left\langle(D h)\left(e_{1}^{2}, e_{2}\right), h\left(e_{1}, e_{2}\right)\right\rangle=\frac{1}{2} e_{1}\left\|h\left(\tilde{e}_{1}, \tilde{e}_{2}\right)\right\|^{2}=\beta e_{1} \beta .
$$

Combining (3.5)-(3.7) we get (3.2)-(3.4).

Now we prove theorem 3.1. Let $M^{2}$ be a surface with constant Gauss curvature $K$, helically immersed in $E^{7}$. If the immersion is of order 1 or 2 , by theorem 2.8, $M^{2}$ is contained in a 5 -dimensional linear subspace of $E^{7}$, thus by the result of Chen and Verheyen $M^{2}$ is of case (i), (ii) or (iii). Suppose the immersion $f$ is of order at least 3 then $K_{1}, K_{2}>0$. Using the notations in [5], i. e. $\alpha=\|H\|, \xi_{3}=(1 / \alpha) H, H$ being the mean curvature vector, $\xi_{4}=1 / 2 \beta\left(h\left(e_{1}^{2}\right)-\right.$ $\left.h\left(e_{2}^{2}\right)\right), \xi_{5}=1 / \beta h\left(e_{1}, e_{2}\right)$, by lemma $3.3(D h)\left(e_{1}^{3}\right)$ is orthogonal to $\xi_{3}, \xi_{4}, \xi_{5}$ since $\beta$ is a constant. We may assume that $\alpha \beta \neq 0$ since the case $\alpha \beta=0$ has been discussed in [5]. But $\left\|(D h)\left(e_{1}^{3}\right)\right\|=K_{1} K_{2}$ so we may assume $(D h)\left(e_{1}^{3}\right)=K_{1} K_{2} \xi_{6}$ then $\xi_{6}$ is a unit vector orthogonal to $\xi_{3}, \xi_{4}$ and $\xi_{5}$. Thus we can find a unit vector $\xi_{7}$ such that $\xi_{3}, \xi_{4}, \xi_{5}, \xi_{6}$ and $\xi_{7}$ form an orthonormal basis for $N_{x} M$. Since $(D h)\left(e_{1}^{2}, e_{2}\right),(D h)\left(e_{1}, e_{2}^{2}\right)$ and $(D h)\left(e_{2}^{3}\right)$ are all orthogonal to $\xi_{3}, \xi_{4}, \xi_{5}$ and $\left\|(D h)\left(e_{2}^{3}\right)\right\|$ $=K_{1} K_{2},\left\langle(D h)\left(e_{1}^{3}\right),(D h)\left(e_{1}^{2}, e_{2}\right)\right\rangle=0,\left\langle(D h)\left(e_{2}^{3}\right),(D h)\left(e_{1}, e_{2}^{2}\right)\right\rangle=0$. We may assume that there are $\theta \in[0,2 \pi]$ and real numbers $a, b$ such that

$$
\begin{aligned}
& (D h)\left(e_{1}^{2}, e_{2}\right)=K_{1} K_{2} a \xi_{7}, \\
& (D h)\left(e_{1}, e_{2}^{2}\right)=K_{1} K_{2} b\left(\cos \theta \xi_{6}-\sin \theta \xi_{7}\right) \\
& (D h)\left(e_{2}^{3}\right)=K_{1} K_{2}\left(\sin \theta \xi_{6}+\cos \theta \xi_{7}\right) .
\end{aligned}
$$

Using lemma 1.1 to $\left\langle(D h)\left(e^{3}\right),(D h)\left(e^{3}\right)\right\rangle=K_{1}^{2} K_{2}^{2}(\langle e, e\rangle)^{3}$ for all $e \in T_{x} M$,

$$
\begin{aligned}
& 2\left\langle(D h)\left(e_{1}^{3}\right),(D h)\left(e_{1}, e_{2}^{2}\right)\right\rangle+3\left\langle(D h)\left(e_{1}^{2}, e_{2}\right),(D h)\left(e_{1}^{2}, e_{2}\right)\right\rangle=K_{1}^{2} K_{2}^{2}, \\
& \left\langle(D h)\left(e_{1}^{3}\right),(D h)\left(e_{2}^{3}\right)\right\rangle+9\left\langle(D h)\left(e_{1}^{2}, e_{2}\right),(D h)\left(e_{1}, e_{2}^{2}\right)\right\rangle=0, \\
& 2\left\langle(D h)\left(e_{1}^{2}, e_{2}\right),(D h)\left(e_{2}^{3}\right)\right\rangle+3\left\langle(D h)\left(e_{1}, e_{2}^{2}\right),(D h)\left(e_{1}, e_{2}^{2}\right)\right\rangle=K_{1}^{2} K_{2}^{2} .
\end{aligned}
$$


Combining all the equations (3.8)-(3.13) we have four solutions :

Case 1. $b=-1, a=-1, \theta=0$; Case 2. $b=1 / 3, a=1 / 3, \theta=0$; Case 3. $b=a=1$, $\theta=\pi$; Case 4. $b=a=-1 / 3, \theta=\pi$. If we replace $\xi_{7}$ by $-\xi_{7}$ then Case 3 reduces to Case 1 and Case 4 reduces to Case 2 . Thus we have basically two possible cases:

$$
\text { Case 1: }(D h)\left(e_{1}^{3}\right)=K_{1} K_{2} \xi_{6}, \quad(D h)\left(e_{1}^{2}, e_{2}\right)=-K_{1} K_{2} \xi_{7} \text {, }
$$

$$
(D h)\left(e_{1}, e_{2}^{2}\right)=-K_{1} K_{2} \xi_{6}, \quad(D h)\left(e_{2}^{3}\right)=K_{1} K_{2} \xi_{7} .
$$

Case 2: $(D h)\left(e_{1}^{3}\right)=K_{1} K_{2} \xi_{6}, \quad(D h)\left(e_{1}^{2}, e_{2}\right)=\left(\frac{1}{3}\right) K_{1} K_{2} \xi_{7}$,

$$
(D h)\left(e_{1}, e_{2}^{2}\right)=\left(\frac{1}{3}\right) K_{1} K_{2} \xi_{6}, \quad(D h)\left(e_{2}^{3}\right)=K_{1} K_{2} \xi_{7} .
$$

We first consider case 2. Choose $\left\{e_{1}, e_{2}\right\}$ to be orthonormal vector fields. Then $\left\{e_{1}, e_{2}, \xi_{3}, \xi_{4}, \xi_{5}, \xi_{6}, \xi_{7}\right\}$ is a moving frame of $E^{7}$. Let $\omega_{1}^{2}$ be the connection form. Then

$$
\begin{aligned}
\nabla_{e_{1}}^{+} \xi_{3} & =\nabla_{e_{1}}^{\perp} \frac{1}{2 \alpha}\left(h\left(e_{1}^{2}\right)+h\left(e_{2}^{2}\right)\right) \\
& =\frac{1}{2 \alpha}\left[(D h)\left(e_{1}^{3}\right)+(D h)\left(e_{1}, e_{2}^{2}\right)+2 \omega_{1}^{2}\left(e_{1}\right) h\left(e_{1}, e_{2}\right)+2 \omega_{2}^{1}\left(e_{1}\right) h\left(e_{1}, e_{2}\right)\right] \\
& =\frac{1}{2 \alpha}\left[K_{1} K_{2} \xi_{6}+\frac{1}{3} K_{1} K_{2} \xi_{6}\right]=\frac{2}{3 \alpha} K_{1} K_{2} \xi_{6}, \\
\nabla_{e_{2}}^{\perp} \xi_{3} & =\nabla_{e_{2}}^{1} \frac{1}{2 \alpha}\left[h\left(e_{1}^{2}\right)+h\left(e_{2}^{2}\right)\right]=\frac{2}{3 \alpha} K_{1} K_{2} \xi_{7} .
\end{aligned}
$$

Thus we have

$$
\nabla^{\perp} \xi_{3}=\frac{2}{3 \alpha} K_{1} K_{2}\left(\omega^{1} \xi_{6}+\omega^{2} \xi_{7}\right)
$$

Similarly we have

$$
\begin{aligned}
& \nabla^{\perp} \xi_{4}=2 \omega_{1}^{2} \xi_{5}+\frac{K_{1} K_{2}}{3 \beta}\left(\omega^{1} \xi_{6}-\omega^{2} \xi_{7}\right), \\
& \nabla^{\perp} \xi_{5}=2 \omega_{2}^{1} \xi_{4}+\frac{K_{1} K_{2}}{3 \beta}\left(\omega^{2} \xi_{6}+\omega^{1} \xi_{7}\right) .
\end{aligned}
$$

The Ricci equation (1.4) can be rewritten as following:

$$
\begin{aligned}
R^{\perp}(X, Y) \xi_{x} & =\nabla_{X}^{\perp} \nabla_{\bar{Y}}^{\frac{1}{Y}} \xi_{x}-\nabla_{\frac{1}{Y}}^{1} \nabla_{X}^{\perp} \xi_{x}-\nabla_{\complement X, Y]}^{\perp} \xi_{x} \\
& =\nabla_{X}^{\perp}\left(\sum_{y} w_{x}^{y}(Y) \xi_{y}\right)-\nabla_{Y}^{1}\left(\sum_{y} \omega_{x}^{y}(X) \xi_{y}\right)-\sum_{y} \omega_{x}^{y}([X, Y]) \xi_{y} \\
& =\sum_{y}\left(X \omega_{x}^{y}(Y)\right) \xi_{y}+\sum_{y} \omega_{x}^{y}(Y) \omega_{y}^{2}(X) \xi_{z}-\sum_{y}\left(Y \omega_{x}^{y}(X)\right) \xi_{y}
\end{aligned}
$$


HELICAL IMMERSIONS AND NORMAL SECTIONS

So we can write

$$
\begin{gathered}
-\sum_{y, z} \omega_{x}^{y}(X) \omega_{y}^{z}(Y) \xi_{z}-\sum_{y} \omega_{x}^{y}([X, Y]) \xi_{y} \\
=\sum_{y}\left[\left(X \omega_{x}^{y}(Y)\right)-\left(Y \omega_{x}^{y}(X)\right)-\omega_{x}^{y}([X, Y])\right. \\
\left.+\sum_{z}\left(\omega_{z}^{y}(X) \omega_{x}^{z}(Y)-\omega_{z}^{y}(Y) \omega_{x}^{z}(X)\right)\right] \xi_{y} \\
=2 \sum_{y}\left(d \omega_{x}^{y}(X, Y)+\sum_{z}\left(\omega_{z}^{y} \wedge \omega_{x}^{z}\right)(X, Y)\right) \xi_{y} .
\end{gathered}
$$

$$
d \omega_{x}^{y}+\sum_{z} \omega_{z}^{y} \wedge \omega_{x}^{z}=\frac{1}{2}\left[A_{\xi_{x}}, A_{\xi_{y}}\right]
$$

where $\left[A_{\xi_{x}}, A_{\xi_{y}}\right]$ denotes a 2 -form, having $\left\langle\left[A_{\xi_{x}}, A_{\xi_{y}}\right](X), Y\right\rangle$ as its value at $X, Y$. Let $x=6, y=7$ then

$$
d \omega_{6}^{7}+\omega_{3}^{7} \wedge \omega_{6}^{3}+\omega_{4}^{7} \wedge \omega_{6}^{4}+\omega_{5}^{7} \wedge \omega_{6}^{5}=\frac{1}{2}\left[A_{\xi_{6}}, A_{\xi_{7}}\right]=0 .
$$

On the other hand

$$
\begin{aligned}
\left\langle\nabla_{e_{1}}^{\perp} \xi_{6}, \xi_{7}\right\rangle & =\left\langle\nabla_{e_{1}}^{\perp}\left(\frac{1}{K_{1} K_{2}}(D h)\left(e_{1}^{3}\right)\right), \frac{3}{K_{1} K_{2}}(D h)\left(e_{1}^{2}, e_{2}\right)\right\rangle \\
& =\frac{3}{K_{1}^{2} K_{2}^{2}}\left\langle\left(D^{2} h\right)\left(e_{1}^{4}\right)+3 \omega_{1}^{2}\left(e_{1}\right)(D h)\left(e_{1}^{2}, e_{2}\right),(D h)\left(e_{1}^{2}, e_{2}\right)\right\rangle \\
& =\frac{9}{K_{1}^{2} K_{2}^{2}} \cdot \frac{K_{1}^{2} K_{2}^{2}}{9} \cdot \omega_{1}^{2}\left(e_{1}\right)=\omega_{1}^{2}\left(e_{1}\right), \\
\left\langle\nabla_{e_{2}}^{1} \xi_{6}, \xi_{7}\right\rangle & =-\left\langle\nabla_{e_{2}}^{1} \xi_{7}, \xi_{6}\right\rangle=-\left\langle\nabla_{e_{2}}^{\perp}\left(\frac{1}{K_{1} K_{2}}(D h)\left(e_{2}^{3}\right)\right), \frac{3}{K_{1} K_{2}}(D h)\left(e_{1}, e_{2}^{2}\right)\right\rangle \\
& =-\omega_{2}^{1}\left(e_{2}\right)=\omega_{1}^{2}\left(e_{2}\right) .
\end{aligned}
$$

Thus $\omega_{6}^{7}=\omega_{1}^{2}$ and (3.16)-(3.20) gives

$$
\begin{aligned}
& d \omega_{1}^{2}+ \frac{2 K_{1} K_{2}}{3 \alpha} \omega^{2} \wedge\left(-\frac{2}{3 \alpha} K_{1} K_{2} \omega^{1}\right)+\left(\frac{K_{1} K_{2}}{\beta} \omega^{2}\right) \wedge\left(-\frac{K_{1} K_{2}}{3 \beta} \omega^{1}\right) \\
&+\left(\frac{K_{1} K_{2}}{3 \beta} \omega^{1}\right) \wedge\left(-\frac{K_{1} K_{2}}{3 \beta} \omega^{2}\right) \\
&=\left(K+\frac{2 K_{1}^{2} K_{2}^{2}}{9 \beta^{2}}-\frac{4 K_{1}^{2} K_{2}^{2}}{9 \alpha^{2}}\right) \omega^{2} \wedge \omega^{1}=\left(1+\frac{2 K_{1}^{2} K_{2}^{2}}{9 \alpha^{2} \beta^{2}}\right) K \omega^{2} \wedge \omega^{1}=0
\end{aligned}
$$

Thus we have $K=0$. Let $x=4, y=5$ then

$$
\begin{aligned}
& d \omega_{4}^{5}+\omega_{3}^{5} \wedge \omega_{4}^{3}+\omega_{6}^{5} \wedge \omega_{4}^{6}+\omega_{7}^{5} \wedge \omega_{4}^{7} \\
& =2 d \omega_{1}^{2}+\left(-\frac{K_{1} K_{2}}{3 \beta} \omega^{2}\right) \wedge\left(\frac{K_{1} K_{2}}{3 \beta} \omega^{1}\right)+\left(-\frac{K_{1} K_{2}}{3 \beta} \omega^{1}\right) \wedge\left(-\frac{K_{1} K_{2}}{3 \beta} \omega^{2}\right) \\
& =\left(2 K-\frac{2 K_{1} K_{2}}{9 \beta^{2}}\right) \omega^{2} \wedge \omega^{1}=-\frac{2 K_{1} K_{2}}{9 \beta^{2}} \omega^{2} \wedge \omega^{1} .
\end{aligned}
$$


On the other hand $\left[A_{\xi_{4}}, A_{\xi_{5}}\right]=4 \beta^{2} \omega^{2} \wedge \omega^{1}$, this is a contradiction. Thus case 2 is impossible.

Next we consider case 1 . Similar computation as in case 2 we have

$$
\begin{aligned}
& \nabla^{\perp} \xi_{3}=0, \\
& \nabla^{\perp} \xi_{4}=2 \omega_{1}^{2} \xi_{5}+\frac{K_{1} K_{2}}{\beta}\left(\omega^{1} \xi_{6}-\omega^{2} \xi_{7}\right), \\
& \nabla^{\perp} \xi_{5}=2 \omega_{2}^{1} \xi_{4}-\frac{K_{1} K_{2}}{\beta}\left(\omega^{2} \xi_{6}+\omega^{1} \xi_{7}\right),
\end{aligned}
$$

and

$$
\begin{aligned}
\left\langle\nabla_{e_{1}}^{\perp} \xi_{6}, \xi_{7}\right\rangle & =\left\langle\nabla_{e_{1}}^{\perp}\left(\frac{1}{K_{1} K_{2}}(D h)\left(e_{1}^{3}\right)\right), \frac{-1}{K_{1} K_{2}}(D h)\left(e_{1}^{2}, e_{2}\right)\right\rangle \\
& =-\frac{1}{K_{1}^{2} K_{2}^{2}}\left\langle\left(D^{2} h\right)\left(e_{1}^{4}\right)+3 \omega_{1}^{2}\left(e_{1}\right)(D h)\left(e_{1}^{2}, e_{2}\right),(D h)\left(e_{1}^{2}, e_{2}\right)\right\rangle \\
& =-3 \omega_{1}^{2}\left(e_{1}\right) .
\end{aligned}
$$

Similarly,

Thus we have

$$
\left\langle\nabla_{e_{2}}^{\perp} \xi_{6}, \xi_{7}\right\rangle=-3 \omega_{1}^{2}\left(e_{2}\right) .
$$

$$
\begin{aligned}
& \nabla^{1} \xi_{6}=-\frac{K_{1} K_{2}}{\beta} \omega^{1} \xi_{4}+\frac{K_{1} K_{2}}{\beta} \omega^{2} \xi_{5}-3 \omega_{1}^{2} \xi_{7} \\
& \nabla^{\perp} \xi_{7}=\frac{K_{1} K_{2}}{\beta} \omega^{2} \xi_{4}+\frac{K_{1} K_{2}}{\beta} \omega^{1} \xi_{5}-3 \omega_{2}^{1} \xi_{6} .
\end{aligned}
$$

Putting $x=4, y=5$ in (3.19)

$$
\begin{aligned}
& d \omega_{4}^{5}+\omega_{6}^{5} \wedge \omega_{4}^{6}+\omega_{7}^{5} \wedge \omega_{4}^{7} \\
& \quad=2 d \omega_{1}^{2}+\frac{K_{1} K_{2}}{\beta} \omega^{2} \wedge \frac{K_{1} K_{2}}{\beta} \omega^{1}+\frac{K_{1} K_{2}}{\beta} \omega^{1} \wedge\left(-\frac{K_{1} K_{2}}{\beta} \omega^{2}\right) \\
& =\left(2 K+\frac{2 K_{1}^{2} K_{2}^{2}}{\beta^{2}}\right) \omega^{2} \wedge \omega^{1}=4 \beta^{2} \omega^{2} \wedge \omega^{1} .
\end{aligned}
$$

Thus we have

$$
K+\frac{K_{1}^{2} K_{2}^{2}}{\beta^{2}}=2 \beta^{2}
$$

Putting $x=6, y=7$ in (3.19)

$$
\begin{aligned}
& d \omega_{6}^{7}+\omega_{4}^{7} \wedge \omega_{6}^{4}+\omega_{5}^{7} \wedge \omega_{6}^{5} \\
& =-3 d \omega_{1}^{2}+\left(-\frac{K_{1} K_{2}}{\beta} \omega^{2}\right) \wedge\left(-\frac{K_{1} K_{2}}{\beta} \omega^{1}\right)+\left(-\frac{K_{1} K_{2}}{\beta} \omega^{1}\right) \wedge\left(\frac{K_{1} K_{2}}{\beta} \omega^{2}\right) \\
& =\left(-3 K+\frac{2 K_{1}^{2} K_{2}^{2}}{\beta^{2}}\right) \omega^{2} \wedge \omega^{1}=0 .
\end{aligned}
$$


Thus we have $-3 K+\left(2 K_{1}^{2} K_{2}^{2}\right) / \beta^{2}=0$. Taking account of (3.26) we have

$$
K_{1}^{2}=\frac{17}{2} K, \quad \beta^{2}=\frac{5}{2} K, \quad K_{2}^{2}=\frac{15}{34} K .
$$

Let $\gamma$ be the geodesic issued from $x \in M$ and $\sigma=f \circ \gamma$ as its image in $E^{\tau}$ Choosing $e_{1}$ such that $e_{1}=\dot{\sigma}$ along $\gamma$,

So

$$
\tilde{\nabla}_{e_{1}} \dot{\sigma}=h\left(e_{1}, e_{1}\right)=\alpha \xi_{3}+\beta \xi_{4}=K_{1} \sigma^{(2)} .
$$

$$
\begin{gathered}
\sigma^{(2)}=\frac{1}{K_{1}}\left(\alpha \xi_{3}+\beta \xi_{4}\right), \\
\tilde{\nabla}_{e_{1}} \sigma^{(2)}=-A_{\sigma}(2) e_{1}+\frac{1}{K_{1}}(D h)\left(e_{1}^{3}\right)=-K_{1} e_{1}+K_{2} \xi_{6}, \quad \text { so } \sigma^{(3)}=\xi_{6}, \\
\tilde{\nabla}_{e_{1}} \sigma^{(3)}=\tilde{\nabla} e_{1} \xi_{6}=\frac{-K_{1} K_{2}}{\beta} \xi_{4}=-K_{2} \sigma^{(2)}+K_{3} \sigma^{(4)}
\end{gathered}
$$

Thus we have $K_{3} \sigma^{(4)}=-\left(\left(K_{1} K_{2}\right) / \beta\right) \xi_{4}+\left(K_{2} / K_{1}\right)\left(\alpha \xi_{3}+\beta \xi_{4}\right)=\left(K_{2} \alpha / K_{1} \beta\right)\left(\beta \xi_{3}-\alpha \xi_{4}\right)$ and

$$
K_{3}=\frac{K_{2} \alpha}{\beta}=\frac{3 \sqrt{2}}{17} \sqrt{ } K
$$

and $\tilde{\nabla}_{e_{1}} \sigma^{(4)}=-K_{3} \xi_{6}=-K_{3} \sigma^{(3)}$. Thus $\gamma$ is a helix of order 4 . But $e_{1}$ can be chosen as any unit vector in $T_{x} M$, this means $f$ is of order 4.

Now if we regard $\left\{\xi_{3}, \xi_{4}, \xi_{5}, \xi_{6}, \xi_{7}\right\}$ as a 5 -plane bundle $E$ on $M^{2}$, then (3.21)-(3.25) define a connection on $E$, equipped with the second fundamental form $h$ and associated second fundamental tensor $A$. It is easy to check that they satisfy the equations of Gauss, Ricci, and Codazzi. Thus by the fundamental theorem of submanifold [2] we can conclude that there is an immersion $M^{2} \rightarrow E^{7}$ with normal bundle $E$, and up to a motion, this immersion is unique.

We can also write this immersion explicitly. Let $e_{1}, e_{2}, \xi_{3}, \xi_{4}, \xi_{5}, \boldsymbol{\xi}_{6}, \boldsymbol{\xi}_{7}$ be the frame at $x, \gamma_{e}$ be the geodesic issued from $x$, having tangent vector $l_{L} e=$ $e_{1} \cos \theta+e_{2} \sin \theta, 0 \leqq \theta<2 \pi, \sigma_{e}=f_{\circ} \gamma_{e}$. Then

$$
\begin{aligned}
& \sigma_{e}^{(1)}(0)=e=e_{1} \cos \theta+e_{2} \sin \theta, \\
& \sigma_{e}^{(2)}(0)=\left(1 / K_{1}\right) h(e, e)=\left(1 / K_{1}\right)\left(\alpha \xi_{3}+\beta \cos 2 \theta \xi_{4}+\beta \sin 2 \theta \xi_{5}\right), \\
& \sigma_{e}^{(3)}(0)=\left(1 / K_{1} K_{2}\right)(D h)\left(e^{3}\right)=\xi_{6} \cos 3 \theta-\xi_{7} \sin 3 \theta, \\
& \sigma_{4}^{(4)}(0)=\left(1 / K_{3}\right)\left(\tilde{\nabla}_{e} \sigma_{e}^{(3)}+K_{2} \sigma_{2}^{(2)}\right)=\left(1 / K_{1}\right)\left(\beta \xi_{3}-\alpha \cos 2 \theta \xi_{4}-\alpha \sin 2 \theta \xi_{5}\right) .
\end{aligned}
$$

Since $\sigma_{e}^{(1)}, \sigma_{e}^{(2)}, \sigma_{e}^{(3)}$ and $\sigma_{e}^{(4)}$ satisfy the Frenet equations and the initial condition (3.29), by solving these equations we get the helical immersion of the sphere $S^{2} \subset E^{7}$, which is the 3-rd standard immersion of $S^{2} \subset E^{7}$ : 


$$
\begin{aligned}
f(\theta, v)= & (R / 16)(\sin v+5 \sin 3 v)\left(e_{1} \cos \theta+e_{2} \sin \theta\right) \\
& -(R \sqrt{6} / 48)(3 \cos v+5 \cos 3 v) \xi_{3} \\
& +(R \sqrt{10} / 16)(\cos v-\cos 3 v)\left(\xi_{4} \cos 2 \theta+\xi_{5} \sin 2 \theta\right) \\
& -(R \sqrt{15} / 16)(\sin v-1 / 3 \sin 3 v)\left(\xi_{6} \cos 3 \theta-\xi_{7} \sin 3 \theta\right),
\end{aligned}
$$

where $R=1 / \sqrt{K}$ is the radius of $S^{2}$ and $(\theta, v)$ is the spherical coordinate on $S^{2}$. Thus Theorem 3.1 is proved.

Now we turn to theorem 3.2 .

LEMMA 3.4. Let $f: M^{2} \subset E^{m}$ be a helical immersion of order $3 . \quad\left\{e_{1}, e_{2}\right\}$ is an orthonormal basis for $T_{x} M, x \in M$. Then

$$
\begin{aligned}
\left(D^{2} h\right)\left(e_{1}^{4}\right)=- & K_{2}^{2} h\left(e_{1}^{2}\right), \\
\left(D^{2} h\right)\left(e_{1}^{3}, e_{2}\right)= & (1 / 2)\left(K_{1}^{2}-K_{2}^{2}-4 \beta^{2}\right) h\left(e_{1}, e_{2}\right), \\
\left(D^{2} h\right)\left(e_{2}, e_{1}^{3}\right)= & (-1 / 2)\left(3 K_{1}^{2}+K_{2}^{2}-12 \beta^{2}\right) h\left(e_{1}, e_{2}\right), \\
\left(D^{2} h\right)\left(e_{1}^{2}, e_{2}^{2}\right)= & \left((-1 / 6) K_{2}^{2}-(1 / 2) K_{1}^{2}+2 \beta^{2}\right) h\left(e_{1}^{2}\right) \\
& +\left((-1 / 6) K_{2}^{2}+(1 / 2) K_{1}^{2}-2 \beta^{2}\right) h\left(e_{2}^{2}\right) .
\end{aligned}
$$

Proof. Let $\gamma$ be a geodesic issued from $x$ with tangent vector $e$ and the Frenet frame for $\sigma=f \circ \gamma$ be $\sigma^{(1)}, \sigma^{(2)}, \sigma^{(3)}$. By theorem 2.1, $\sigma^{(1)}=e, \sigma^{(2)}=$ $\left(1 / K_{1}\right) h\left(e^{2}\right), \sigma^{(3)}=\left(K_{1} K_{2}\right)^{-1}(D h)\left(e^{3}\right)$. And the Frenet formula gives $\tilde{\nabla}_{e} \sigma^{(3)}=-K_{2} \sigma^{(2)}$, i.e.

$$
\left(K_{1} K_{2}\right)^{-1}\left(D^{2} h\right)\left(e^{4}\right)=\left(-K_{2} \sqrt{K_{1}}\right) h\left(e^{2}\right),
$$

or

$$
\left(D^{2} h\right)\left(e^{4}\right)=-K_{2}^{2} h\left(e^{2}\right) .
$$

Since this is true for all unit vectors $e \in U_{x}(M)$ by lemma 1.1 we have

$$
\begin{aligned}
& 3\left(D^{2} h\right)\left(e_{1}^{3}, e_{2}\right)+\left(D^{2} h\right)\left(e_{2}, e_{1}^{3}\right)=-2 K_{2}^{2} h\left(e_{1}, e_{2}\right), \\
& \left(D^{2} h\right)\left(e_{1}^{2}, e_{2}^{2}\right)+\left(D^{2} h\right)\left(e_{2}^{2}, e_{1}^{2}\right)=-\left(\frac{1}{3}\right) K_{2}^{2}\left(h\left(e_{1}^{2}\right)+h\left(e_{2}^{2}\right)\right) .
\end{aligned}
$$

By the Ricci identity

$$
\left(D^{2} h\right)\left(e_{1}^{3}, e_{2}\right)-\left(D^{2} h\right)\left(e_{2}, e_{1}^{3}\right)=R^{\perp}\left(e_{1}, e_{2}\right) h\left(e_{1}^{2}\right)-2 h\left(R\left(e_{1}, e_{2}\right) e_{1}, e_{1}\right) .
$$

Using (1.3) and Proposition 13 in [5] there is an adapted orthonormal frame $\left\{e_{1}, e_{2}, \xi_{3}, \cdots, \xi_{m}\right\}$ for which we can find that

$$
R^{\perp}\left(e_{1}, e_{2}\right) \xi_{2}=0 \text { if } i \neq 4,5 ; R^{\perp}\left(e_{1}, e_{2}\right) \xi_{4}=-2 \beta^{2} \xi_{5} ; R^{\perp}\left(e_{1}, e_{2}\right) \xi_{5}=2 \beta^{2} \xi_{4},
$$

since $R\left(e_{1}, e_{2}\right) e_{1}=-K e_{2}=\left(-K_{1}^{2}+3 \beta^{2}\right) e_{2}$, where $K$ is the Gauss curvature of $M^{2}$. Thus we have 


$$
\left(D^{2} h\right)\left(e_{1}^{3}, e_{2}\right)-\left(D^{2} h\right)\left(e_{2}, e_{1}^{3}\right)=2\left(K_{1}^{2}-4 \beta^{2}\right) h\left(e_{1}, e_{2}\right) .
$$

By (3.34) and (3.37) we get (3.31) and (3.32).

Similarly we have

$$
\begin{aligned}
& \left(D^{2} h\right)\left(e_{1}^{2}, e_{2}^{2}\right)-\left(D^{2} h\right)\left(e_{2}^{2}, e_{1}^{2}\right) \\
& \quad=R^{1}\left(e_{1}, e_{2}\right) h\left(e_{1}, e_{2}\right)-h\left(R\left(e_{1}, e_{2}\right) e_{1}, e_{2}\right)-h\left(R\left(e_{1}, e_{2}\right) e_{2}, e_{1}\right) \\
& =\beta^{2}\left[h\left(e_{1}^{2}\right)-h\left(e_{2}^{2}\right)\right]+\left(K_{1}^{2}-3 \beta^{2}\right) h\left(e_{2}^{2}\right)-\left(K_{1}^{2}-3 \beta^{2}\right) h\left(e_{1}^{2}\right) \\
& =-\left(K_{1}^{2}-4 \beta^{2}\right)\left[h\left(e_{1}^{2}\right)-h\left(e_{2}^{2}\right)\right] .
\end{aligned}
$$

Taking into account of (3.35) we get (3.33).

LEMMA 3.5. Let $f: M^{2} \rightarrow E^{m}$ be a helical immersion of order 3 . Then $M^{2}$ has constant Gauss curvature.

Proof. Suppose $M^{2}$ is not of constant Gauss curvature then $\beta$ is not a constant. Since $M^{2}$ is connected there exists $x \in M^{2}$ such that $\beta \neq 0, d \beta \neq 0$ in a neighborhood $U$ of $x$. Choose a unit vector field $e_{1}$ in $U$ such that $d \beta\left(e_{1}\right)=$ $e_{1} \beta=0$ and a unit vector field $e_{2}$ in $U$ orthogonal to $e_{1}$. Then by Lemma 3.3

$$
\left\langle(D h)\left(e_{1}^{3}\right), h\left(e_{2}^{2}\right)\right\rangle=0 .
$$

Differentiating along the direction of $e_{2}$ we have

$$
\begin{aligned}
& \left\langle\left(D^{2} h\right)\left(e_{2}, e_{1}^{3}\right), h\left(e_{2}^{2}\right)\right\rangle+\left\langle(D h)\left(e_{1}^{3}\right),(D h)\left(e_{2}^{3}\right)\right\rangle \\
& \quad+3 \omega_{1}^{2}\left(e_{2}\right)\left\langle(D h)\left(e_{1}^{2}, e_{2}\right), h\left(e_{2}^{2}\right)\right\rangle+2 \omega_{2}^{1}\left(e_{2}\right)\left\langle(D h)\left(e_{1}^{3}\right), h\left(e_{1}, e_{2}\right)\right\rangle=0 .
\end{aligned}
$$

By lemma 3.3 and 3.4 we get

$$
\left\langle(D h)\left(e_{1}^{3}\right),(D h)\left(e_{2}^{3}\right)\right\rangle=-3 \omega_{1}^{2}\left(e_{2}\right)\left\langle(D h)\left(e_{1}^{2}, e_{2}\right) ; h\left(e_{2}^{2}\right)\right\rangle=3 \omega_{1}^{2}\left(e_{2}\right) \beta e_{2} \beta
$$

Also by Lemma 3.3

$$
\left\langle(D h)\left(e_{1}, e_{2}^{2}\right), h\left(e_{1}^{2}\right)\right\rangle=0 .
$$

Differentiating along the direction of $e_{2}$ we have

$$
\begin{aligned}
& \left\langle(D h)\left(e_{1}, e_{2}^{2}\right),(D h)\left(e_{1}^{2}, e_{2}\right)\right\rangle+\left\langle\left(D^{2} h\right)\left(e_{2}^{3}, e_{1}\right), h\left(e_{1}^{2}\right)\right\rangle+\omega_{1}^{2}\left(e_{2}\right)\left\langle(D h)\left(e_{2}^{3}\right), h\left(e_{1}^{2}\right)\right\rangle \\
& \quad+2 \omega_{2}^{1}\left(e_{2}\right)\left\langle(D h)\left(e_{1}^{2}, e_{2}\right), h\left(e_{1}^{2}\right)\right\rangle+2 \omega_{1}^{2}\left(e_{2}\right)\left\langle(D h)\left(e_{1}, e_{2}^{2}\right), h\left(e_{1}, e_{2}\right)\right\rangle=0 .
\end{aligned}
$$

Hence we have

that is

$$
\left\langle(D h)\left(e_{1}, e_{2}^{2}\right),(D h)\left(e_{1}^{2}, e_{2}\right)\right\rangle+\omega_{1}^{2}\left(e_{2}\right)\left(-3 \beta e_{2} \beta\right)+2 \omega_{1}^{2}\left(e_{2}\right)\left(\beta e_{2} \beta\right)=0,
$$

$$
\left\langle(D h)\left(e_{1}, e_{2}^{2}\right),(D h)\left(e_{1}^{2}, e_{2}\right)\right\rangle=\omega_{1}^{2}\left(e_{2}\right) \beta e_{2} \beta .
$$

Combining (3.38), (3.39) and (3.12) we find 


$$
\begin{gathered}
\left\langle(D h)\left(e_{1}^{3}\right),(D h)\left(e_{2}^{3}\right)\right\rangle=\left\langle(D h)\left(e_{1}, e_{2}^{2}\right),(D h)\left(e_{1}^{2}, e_{2}\right)\right\rangle=0, \\
\omega_{1}^{2}\left(e_{2}\right) \beta e_{2} \beta=0 .
\end{gathered}
$$

(3.41) is true for all points in $U$. But $\beta e_{2} \beta \neq 0$ in $U$, thus $\omega_{1}^{2}\left(e_{2}\right)=0$ in $U$.

Again, since $(D h)\left(e_{1}^{3}\right)$ is orthogonal to $h\left(e_{1}^{2}\right), h\left(e_{1}, e_{2}\right)$ and $h\left(e_{2}^{2}\right)$, so by (3.36), we have

$$
R^{\perp}\left(e_{1}, e_{2}\right)(D h)\left(e_{1}^{3}\right)=0 \text {. }
$$

By (3.30)-(3.33), we have

$$
\begin{aligned}
\nabla_{e_{1}}^{\perp} \nabla_{e_{2}}^{\perp}(D h)\left(e_{1}^{3}\right) & =\nabla_{e_{1}}^{\perp}\left(D^{2} h\right)\left(e_{2}, e_{1}^{3}\right) \\
& =-\frac{1}{2}\left(3 K_{1}^{2}+K_{2}^{2}-12 \beta^{2}\right) \nabla_{e_{1}}^{\perp} h\left(e_{1}, e_{2}\right) \\
& =-\frac{1}{2}\left(3 K_{1}^{2}+K_{2}^{2}-12 \beta^{2}\right)\left[(D h)\left(e_{1}^{2}, e_{2}\right)+\omega_{1}^{2}\left(e_{1}\right) h\left(e_{2}^{2}\right)+\omega_{2}^{1}\left(e_{1}\right) h\left(e_{1}^{2}\right)\right], \\
\nabla_{e_{2}}^{\perp} \nabla_{e_{1}}^{\perp}(D h)\left(e_{1}^{3}\right) & =\nabla_{e_{2}}^{\perp}\left[\left(D^{2} h\right)\left(e_{1}^{4}\right)+3 \omega_{1}^{2}\left(e_{1}\right)(D h)\left(e_{1}^{2}, e_{2}\right)\right] \\
& =\nabla_{e_{2}}^{\perp}\left[-K_{2}^{2} h\left(e_{1}^{2}\right)+3 \omega_{1}^{2}\left(e_{1}\right)(D h)\left(e_{1}^{2}, e_{2}\right)\right] \\
& =-K_{2}^{2}(D h)\left(e_{1}^{2}, e_{2}\right)+3\left[e_{2} \omega_{1}^{2}\left(e_{1}\right)\right](D h)\left(e_{1}^{2}, e_{2}\right)+3 \omega_{1}^{2}\left(e_{1}\right)\left(D^{2} h\right)\left(e_{2}^{2}, e_{1}^{2}\right), \\
\nabla_{\left[e_{1}, e_{2}\right]}^{\perp}(D h)\left(e_{1}^{3}\right) & =\omega_{2}^{1}\left(e_{1}\right) \nabla_{e_{1}}^{\perp}(D h)\left(e_{1}^{3}\right)=\omega_{2}^{1}\left(e_{1}\right)\left[-K_{2}^{2} h\left(e_{1}^{2}\right)+3 \omega_{1}^{2}\left(e_{1}\right)(D h)\left(e_{1}^{2}, e_{2}\right)\right] .
\end{aligned}
$$

Thus,

$$
\begin{aligned}
R^{\perp}\left(e_{1}, e_{2}\right)(D h)\left(e_{1}^{3}\right) & =\nabla_{e_{1}}^{\perp} \nabla_{e_{2}}^{\perp}(D h)\left(e_{1}^{3}\right)-\nabla_{e_{2}}^{\perp} \nabla_{e_{1}}^{\perp}(D h)\left(e_{1}^{3}\right)-\nabla_{\left[e_{1}, e_{2}\right]}^{\perp}(D h)\left(e_{1}^{3}\right) \\
& =\left[-\frac{1}{2}\left(3 K_{1}^{2}+K_{2}^{2}-12 \beta^{2}\right)+K_{2}^{2}-3 K\right](D h)\left(e_{1}^{2}, e_{2}\right) \\
& =-\frac{1}{2}\left(9 K_{1}^{2}-K_{2}^{2}-30 \beta^{2}\right)(D h)\left(e_{1}^{2}, e_{2}\right) .
\end{aligned}
$$

Since $\beta$ is not a constant, we have $(D h)\left(e_{1}^{2}, e_{2}\right)=0$. On the other hand, by differentiating $\left\langle(D h)\left(e_{1}^{2}, e_{2}\right), h\left(e_{1}^{2}\right)\right\rangle=0$ along the direction of $e_{2}$, we have

hence

$$
\left\langle(D h)\left(e_{1}^{2}, e_{2}\right),(D h)\left(e_{1}^{2}, e_{2}\right)\right\rangle+\left\langle(D h)\left(e_{2}^{2}, e_{1}^{2}\right), h\left(e_{1}^{2}\right)\right\rangle=0,
$$

$$
\begin{aligned}
& \left\langle(D h)\left(e_{1}^{2}, e_{2}\right),(D h)\left(e_{1}^{2}, e_{2}\right)\right\rangle=-\left\langle(D h)\left(e_{2}^{2}, e_{1}^{2}\right), h\left(e_{1}^{2}\right)\right\rangle \\
& =\frac{1}{3} K_{1}^{2} K_{2}^{2}-\frac{1}{3} K_{2}^{2} \beta^{2}-K_{1}^{2} \beta^{2}+4 \beta^{4}=0 .
\end{aligned}
$$

This shows that $\beta$ is a constant, a contradiction. This proves Lemma 3.5.

Now we finish the proof of theorem 3.2. Let $M^{2} \subset E^{m}$ be a helical immersion of order 3. By lemma 3.5, $M^{2}$ has constant Gauss curvature. By theorem 2.9 , we may assume that $m=9$. Let $\left\{e_{1}, e_{2}\right\}$ be orthonormal vector fields in some open subset $U \subset M$. Thus Lemma 3.3 shows that $(D h)\left(e_{1}^{3}\right)$ is orthogonal to $h\left(e_{1}^{2}\right), h\left(e_{1}, e_{2}\right)$ and $h\left(e_{2}^{2}\right)$, by (3.36), we have 


$$
R^{\perp}\left(e_{1}, e_{2}\right)(D h)\left(e_{1}^{3}\right)=0 .
$$

The same computation as in the proof of lemma 3.5 gives

$$
-\frac{1}{2}\left(9 K_{1}^{2}-K_{2}^{2}-30 \beta^{2}\right)(D h)\left(e_{1}^{2}, e_{2}\right)=0 \text {. }
$$

But we also have

$$
\left\langle(D h)\left(e_{1}^{2}, e_{2}\right),(D h)\left(e_{1}^{2}, e_{2}\right)\right\rangle=\frac{1}{3} K_{1}^{2} K_{2}^{2}-\frac{1}{3} K_{2}^{2} \beta^{2}-K_{1}^{2} \beta^{2}+4 \beta^{4},
$$

and

$$
\begin{aligned}
\left\langle(D h)\left(e_{1}^{2}, e_{2}\right),(D h)\left(e_{1}^{2}, e_{2}\right)\right\rangle & =-\left\langle\left(D^{2} h\right)\left(e_{1}^{3}, e_{2}\right), h\left(e_{1}, e_{2}\right)\right\rangle \\
& =\frac{1}{2}\left(-K_{1}^{2}+K_{2}^{2}+4 \beta^{2}\right) \beta^{2} .
\end{aligned}
$$

Comparing (3.43) and (3.44), we get

$$
12 \beta^{4}-\left(5 K_{2}^{2}+3 K_{1}^{2}\right) \beta^{2}+2 K_{1}^{2} K_{2}^{2}=0
$$

If $(D h)\left(e_{1}^{2}, e_{2}\right)=0$, by (3.44) we have $\beta=0$ or $-K_{1}^{2}+K_{2}^{2}+4 \beta^{2}=0$, both are impossible by (3.45). If $(D h)\left(e_{1}^{2}, e_{2}\right) \neq 0$, then $9 K_{1}^{2}-K_{2}^{2}-30 \beta^{2}=0$, this also contradicts with (3.45). Thus theorem 3.2 is proved.

Since a helical immersion $M^{2} \subset E^{6}$ has order no more than 3 ([5]), we have the following.

COROLlary 3.6. Let $M^{2}$ be a surface immersed into $E^{6}$. $M^{2}$ has geodesic normal sections if and only if $M^{2}$ is contained on one of the surfaces (i), (ii) and (iii) listed in theorem 3.1.

Acknowlegement. We are grateful to the referee for his valuable suggestions which lead improvements to this paper.

\section{REFERENCES}

[1] Besse, A.L., Manifolds all of whose geodesics are closed, Springer-Verlag, Berlin, 1978.

[2] CHEN, B.Y., Geometry of submanifolds, M. Dekker, New York, 1973.

[3] CHEN, B.Y., Submanifolds with planar normal sections, Soochow J. Math., 7 (1981), 19-24.

[4] CHEN, B.Y., Differential geometry of submanifolds with planar normal sections, Ann. Mat. Pura Appl., Vol. 130 (1982), 59-66.

[5] Chen, B.Y. AND P. Verheyen, Submanifolds with geodesic normal sections, Math. Ann., 269 (1984), 417-429.

[6] Ferus, D. and S. Schirrmacher, Submanifolds in Euclidean spaces with simple geodesics, Math. Ann., 260 (1982), 57-62.

[7] HoNG, Y., Houn, C.S. AND G.Q. WANG, Some surfaces with pointwise planar 
normal sections, Bull. Soc. Math. Belg., 36 (1984), 193-206.

[8] NakAgAwa, H., On a certain minimal immersion of a Riemannian manifold into a sphere, Kodai Math. J., 3 (1980), 321-340.

[9] Sakamoto, K., Helical immersions into a unit sphere, Math. Ann., 261 (1982), 63-80.

[10] Verhayen, P., Submanifolds of $E^{m}$ with geodesic normal sections are helical, to appear.

Department of Mathematics

Wayne State University

Detroit, Michigan 48202

U.S.A. 